\title{
Multi-wavelength observations of Proxima Centauri ${ }^{\star}, \star \star$
}

\author{
B. Fuhrmeister ${ }^{1}$, S. Lalitha ${ }^{1}$, K. Poppenhaeger ${ }^{1}$, N. Rudolf ${ }^{1}$, C. Liefke ${ }^{1,2}$, A. Reiners ${ }^{3}$, \\ J. H. M. M. Schmitt ${ }^{1}$, and J.-U. Ness ${ }^{4}$
}

\author{
1 Hamburger Sternwarte, University of Hamburg, Gojenbergsweg 112, 21029 Hamburg, Germany \\ e-mail: bfuhrmeister@hs.uni-hamburg.de \\ 2 Zentrum für Astronomie, Mönchhofstraße 12-14, 69120 Heidelberg, Germany \\ 3 Institute of Astrophysics, University of Göttingen, Friedrich-Hund-Platz 1, 37077 Göttingen, Germany \\ ${ }^{4}$ XMM-Newton Science Operations Centre, European Space Agency (ESA/ESAC), 28691 Villanueva de la Cañada, Madrid, Spain
}

Received 9 June 2011 / Accepted 18 August 2011

\section{ABSTRACT}

\begin{abstract}
Aims. We report simultaneous observations of the nearby flare star Proxima Centauri with VLT/UVES and XMM-Newton over three nights in March 2009. Our optical and X-ray observations cover the star's quiescent state, as well as its flaring activity and allow us to probe the stellar atmospheric conditions from the photosphere into the chromosphere, and then the corona during its different activity stages.

Methods. Using the X-ray data, we investigate variations in coronal densities and abundances and infer loop properties for an intermediate-sized flare. The optical data are used to investigate the magnetic field and its possible variability, to construct an emission line list for the chromosphere, and use certain emission lines to construct physical models of Proxima Centauri's chromosphere.

Results. We report the discovery of a weak optical forbidden Fe xII line at $3388 \AA$ during the more active states of Proxima Centauri. For the intermediate flare, we find two secondary flare events that may originate in neighbouring loops, and discuss the line asymmetries observed during this flare in $\mathrm{H}_{\mathrm{I}}, \mathrm{He}_{\mathrm{I}}$, and $\mathrm{Ca}$ II lines. The high time-resolution in the $\mathrm{H} \alpha$ line highlights strong temporal variations in the observed line asymmetries, which re-appear during a secondary flare event. We also present theoretical modelling with the stellar atmosphere code PHOENIX to construct flaring chromospheric models.
\end{abstract}

Key words. stars: activity - stars: magnetic field - stars: chromospheres - stars: coronae - stars: late-type stars: individual: Proxima Centauri

\section{Introduction}

Coronal heating of cool stars is thought to be driven by magnetic fields generated in the stellar convection zones. For stars from spectral type $\mathrm{F}$ to mid-M, the magnetic field production process can be modelled with an $\alpha \Omega$ dynamo (Parker 1955). However, stars of spectral type M3 or later are fully convective (Chabrier $\&$ Baraffe 1997; Dorman et al. 1989) and are not expected to undergo the same dynamo process as earlier-type stars (Browning 2008). Other processes such as turbulent or $\alpha^{2}$ dynamos should not be as effective as the $\alpha \Omega$ dynamo and, indeed, many late $\mathrm{M}$ dwarfs have rather low X-ray luminosities in their states of quiescence (Robrade \& Schmitt 2005). Nevertheless, the same stars are capable of producing strong flares of short as well as long durations (Stelzer et al. 2006; Robrade et al. 2010), so there must be some mechanism to allow violent releases of large amounts of magnetic energy. Magnetic flux densities are enormous in mid- to late-M stars, providing firm evidence of an efficient mechanism to produce and maintain strong magnetic fields (e.g. Reiners \& Basri 2007, 2010). In summary, the magnetic activity processes in late $\mathrm{M}$ dwarfs are not yet thoroughly understood in

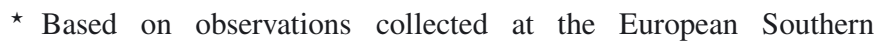
Observatory, Paranal, Chile, 082.D-0953A and on observations obtained with XMM-Newton, an ESA science mission with instruments and contributions directly funded by ESA Member states and NASA.

$\star \star$ Full Table 6 is only available at the CDS via anonymous ftp to cdsarc.u-strasbg.fr $(130.79 .128 .5)$ or via

http://cdsarc.u-strasbg.fr/viz-bin/qcat?J/A+A/534/A133 detail. Multi-wavelength observations cover different activity indicators and can therefore help us to reconstruct a picture of the whole stellar atmosphere. Examples of multi-wavelength observations can be found in e.g. Osten et al. (2005a), Berger et al. (2008), Kowalski et al. (2010a), and Fuhrmeister et al. (2008).

The energy released in flares is thought to originate from the interaction of magnetic fields in the convective zone of the star and photospheric motions that entangle the magnetic loops penetrating the stellar surface. The energy release is triggered by magnetic instabilities that cause the entangled magnetic field lines to reconnect in the corona leading to heating and particle acceleration in the reconnection region. Particles are accelerated downwards into the chromosphere where they collisionally heat the denser plasma, which in turn expands and evaporates into the corona. The coronal loop is filled by dense, hot plasma, which is then observable as a flare in soft X-rays (see e.g. Haisch et al. 1991).

Proxima Centauri, with a distance of only $1.3 \mathrm{pc}$ (van Leeuwen 2007) the star closest to the Sun, is a magnetically active star of spectral type dM5.5. It has been frequently observed by various X-ray satellites: Einstein (Haisch et al. 1980), EXOSAT-IUE (Haisch et al. 1983), XTE (Haisch et al. 1998), ROSAT (Voges et al. 1999), ASCA (Haisch et al. 1995), Chandra (Wargelin \& Drake 2002), and XMM-Newton (Güdel et al. 2004). Its quiescent $X$-ray luminosity varies in the range $L_{\mathrm{X}} \approx(4-16) \times 10^{26} \mathrm{erg} \mathrm{s}^{-1}$, which is comparable to that of the Sun despite its 50 times smaller surface area. Over the past 30 years, several X-ray flares of Proxima Centauri have been 
observed, with the most extreme peak luminosities observed in a 2001 XMM-Newton observation (Güdel et al. 2004), which exceeded typical quiescent state X-ray fluxes by a factor of $\approx 100$. Here we present new data for Proxima Centauri's coronal and chromospheric properties, together with simultaneous measurements of its large-scale magnetic field strength.

Our paper is structured as follows. In Sect. 2, we describe our observations obtained with VLT/UVES and XMM-Newton. In Sect. 3, we compare the timing behaviour of Proxima Centauri in different wavelength bands. The coronal properties of Proxima Centauri such as temperatures and elemental abundances are presented in Sect. 4, while Sect. 5 describes the chromospheric and transition region properties of the star. Sections 6 and 7 contain a discussion of the presented findings and our conclusions.

\section{Observations and data analysis}

The multi-wavelength observations reported in this paper were obtained strictly simultaneously with XMM-Newton and ESO's Kueyen telescope equipped with the Ultraviolet-Visual Echelle Spectrograph (UVES) on 9, 11, and 13 March 2009 (labelled "night 1", "night 2" and "night 3" in the following; see also Table 1). Due to the proximity of Proxima Centauri, interstellar absorption is negligible for the optical as well as for the X-ray data.

\subsection{Optical UVES data}

For our optical observations, the UVES spectrograph was operated in a dichroic mode leading to a spectral coverage from about $3290 \AA$ to $4500 \AA$ in the blue arm and $6400 \AA$ to $10080 \AA$ in the red arm with a small gap from $8190 \AA$ to $8400 \AA$ caused by the CCD mosaic ${ }^{1}$. For the red arm, a non-standard setup was used to ensure coverage of the $\mathrm{H} \alpha$ line. We used exposure times varying from 1000 to $1800 \mathrm{~s}$ for the blue arm and of 90 to $450 \mathrm{~s}$ in the red arm due to variable seeing conditions. In the blue arm, we obtained 24, 17, and 15 useful spectra during the three nights and in the red $\operatorname{arm} 215,168$, and 179 useful spectra, respectively. The typical resolution of our spectra is $\sim 45000$. The red arm spectra were reduced using the UVES pipeline vers. 4.3.0 (Ballester et al. 2000) $)^{2}$. The blue arm spectra could not be properly reduced with the pipeline software but had to be reduced manually using the IDL reduction software REDUCE (Piskunov \& Valenti 2002) for several reasons. To obtain a simultaneous coverage with XMM-Newton, the star had to be observed at rather high airmass starting with a maximum of 2.4. Since the positioning of the star was accomplished with the red arm, a wider slit had to be used for the blue spectra, which resulted in an overlap of the bluest spectral orders. Therefore no dark exposure could be acquired and the flat-field and science spectra extraction had to be carried out with a fixed width and without scattered light. To complicate the spectral reduction even more, quite a number of spectra are contaminated by solar stray-light (from the Moon), which is outshone by the star in the red part of the spectrum but can be clearly recognised in the blue part of the spectrum.

The wavelength calibration in the blue arm was carried out with thorium-argon spectra with an accuracy of $\sim 0.04 \AA$. Since the weather conditions and especially the seeing varied throughout the nights, no absolute flux calibration with a standard star

\footnotetext{
${ }^{1}$ A detailed description of the UVES spectrograph is available at http://www. eso.org/instruments/uves/doc/

2 The UVES pipeline manual can be found at $\mathrm{ftp}$.eso.org/pub/ $\mathrm{dfs} /$ pipelines/uves-cpl/uves-pipeline-manual-13.0.pdf
}

could be performed. To obtain an a posteriori flux calibration, we used synthetic stellar spectra provided by the stellar atmosphere program PHOENIX (Hauschildt et al. 1999). We determined the best-fit stellar model using a grid with $T_{\text {eff }}$ varying from $2700 \mathrm{~K}$ to $3400 \mathrm{~K}$ in steps of $100 \mathrm{~K}$ and $\log g$ of $4.5,5.0$, and 5.5 and found that model to have $T_{\text {eff }}=3100 \mathrm{~K}$ and $\log g=5.5 \mathrm{in}$ good agreement with the values derived by Demory et al. (2009), who had derived $T_{\text {eff }}=3098 \pm 56 \mathrm{~K}$ and $\log g=5.2$ using the VLTI. For the blue spectra, only data at wavelength redward of $4000 \AA$ were used for the calibration since at short wavelengths the data are dominated by chromospheric emission and thus the model spectra are unreliable. We estimate that our errors in the flux calibration in the blue band are about a factor of two. A comparison with a flux-calibrated spectrum of Proxima Centauri acquired by Cincunegui et al. (2007) and Cincunegui \& Mauas (2004) shows that our fluxes are higher by about a factor of two for the blue band and by a factor of two to three in the red band. Since these authors used a standard star and low resolution spectra for their flux calibration, the main source of error in our calibration seems to be the model spectrum.

In addition to the spectral data, we obtained a blue and a red light curve using the UVES exposure-meters, i.e., one blue and red photometer located in the two arms of the spectrograph. These data are normally obtained for engineering purposes only and are not flux calibrated. We note that the flux bands of the exposure-meters are not identical to the spectral bands, the blue flux band in particular being "redder" than the blue spectrum.

\subsection{X-ray data}

Each of our VLT observations was accompanied by three simultaneous $30 \mathrm{ks}$ observations conducted with XMM-Newton; the exact observation times with the ObsIDs being given in Table 1. On board XMM-Newton, there are three telescopes focusing X-rays onto three CCD cameras (one PN and two metal oxide semi-conductor (MOS) cameras with a sensitivity range of $\approx 0.2-15 \mathrm{keV}$ ), which together form the European Photon Imaging Camera (EPIC). The X-ray telescopes with the MOS detectors are also equipped with reflection gratings. The two RGS (Reflection Grating Spectrometers) provide highresolution $\mathrm{X}$-ray spectroscopy $(E / \Delta E \approx 200-800)$ in the energy range of $0.35-2.5 \mathrm{keV}$ capable of resolving individual X-ray emission lines. The X-ray instruments are accompanied by the Optical Monitor (OM), an optical/UV telescope that can be used with different filters for imaging and time-resolved photometry. Useful data of Proxima Centauri were obtained with the OM, EPIC, and RGS detectors, which were all operated simultaneously. The PN and MOS detectors were operated with the medium filter in full frame and large window mode, respectively. The OM was operated in fast mode with $0.5 \mathrm{~s}$ cadence using the $U$ band filter covering a band pass of 300-390 nm.

All XMM-Newton data were reduced using the standard $X M M-N e w t o n$ Science Analysis System (SAS) software, version 10.0. The EPIC light curves and spectra were obtained using standard filtering criteria. We adopted an extraction radius of $15^{\prime \prime}$ centred on the source; for background subtraction we used nearby source-free areas. Source counts with energies in the energy range $0.2-10 \mathrm{keV}$ were considered for the scientific analysis. For the first two nights, the X-ray observations displayed only a low background level. During the third night, short phases with high background levels were present; these time intervals were excluded from our spectral analysis. The X-ray light 
Table 1. The three XMM-Newton observations of Proxima Centauri in March 2009.

\begin{tabular}{lccc}
\hline \hline ObsID & Start time & Duration (ks) & Label \\
\hline 0551120301 & $2009-03-1002: 23: 34$ & 28.7 & night 1 \\
0551120201 & $2009-03-1202: 11: 19$ & 30.7 & night 2 \\
0551120401 & 2009-03-14 02:20:45 & 28.7 & night 3 \\
\hline
\end{tabular}

curves are background-subtracted and binned by $100 \mathrm{~s}$ unless stated otherwise.

Spectral analysis was carried out with Xspec V12.5.0 (Arnaud 1996) for the overall fitting processes and CORA (Ness \& Wichmann 2002) for fitting of individual spectral lines. For the overall analysis in Xspec, we used models with several temperature components assuming the same elemental abundance for each component. These models are based on a collisionally ionised optically thin gas calculated with the APEC code (Smith et al. 2001). Abundances are calculated relative to the solar photospheric values given by Grevesse \& Sauval (1998).

\section{Multi-wavelength timing behaviour}

In Figs. 1-3, we present the background-subtracted X-ray light curves (taken with EPIC-PN) and the OM $U$-band light curve for our three Proxima Centauri observations, as well as the optical UVES blue-band flux and several chromospheric line fluxes; the $\mathrm{X}$-ray light curves are binned in units of $100 \mathrm{~s}$, and data for the $\mathrm{OM}$ in units of $10 \mathrm{~s}$, while the UVES exposure-meter data are binned in units of $5 \mathrm{~s}$.

\subsection{Quiescence and small flares}

In the optical (UVES exposure-meter and in the OM), flaring state and quiescent phases can easily be identified even for smaller flares. For the chromospheric emission line light curves and the X-ray light curves this is not so easy: the lowest parts of the X-ray light curve - as seen in Figs. 1-3 - show a typical count rate of $\approx 1 \mathrm{cps}$ in the $\mathrm{PN}$ detector, comparable to the low count rate states found in the 2001 observations of Proxima Centauri (see Güdel et al. 2002). During these time intervals, the light curves also display some variability, i.e. do not remain constant. To be consistent with other authors, we nevertheless use the term "quiescence" and define it for our subsequent spectral analysis as those times with a PN count rate below $1.8 \mathrm{cps}$.

In addition to the low-level variability seen during quiescence, small flares are observed at 3:30 and 6:00 UT in the X-ray data from night 1 (see Fig. 1). Similarly, in the X-ray data from night 2 (Fig. 2), which have a slightly higher mean count rate, there is some low-level variation with small flares at about 4:40, 6:15 and 8:40 UT. Most of these flares are also visible in the OM light curves, while the UVES exposure-meter does not show significant variability. Apart from these small flares, the X-ray light curves also show some plateau-like structures and slow rises and decreases not seen in the OM light curve, which displays a constant quiescent level during these times.

Nevertheless, a comparison of the X-ray flux with several chromospheric line fluxes (see Figs. 1 and 2) shows quite good agreement, especially for the $\mathrm{H} \alpha$ light curve, which can be studied with the much higher time-resolution of the red arm spectra. For instance, the first $\mathrm{H} \alpha$ flare in night 1 at about 3:30 UT can also be identified in the X-ray light curve, although not in the optical (UVES) light curve. In addition, the flare in night 2 at 6:15 UT is found in X-ray, OM, and in the chromospheric

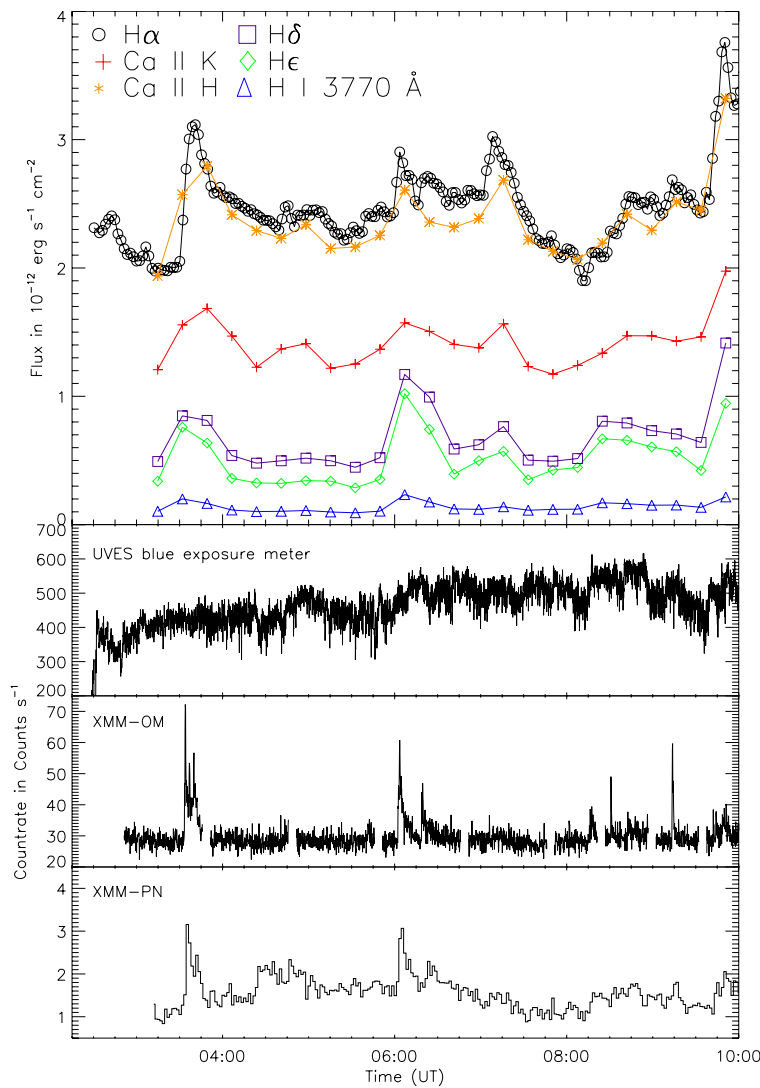

Fig. 1. Light curves of Proxima Centauri from night 1 as seen in the fluxes of characteristic chromospheric emission lines (top, note the better time binning provided by the $\mathrm{H} \alpha$ line) as well as optical UVES, OM, and X-ray light curves (bottom).

emission lines, but is much more pronounced in the $\mathrm{Ca}$ II $\mathrm{H} \& \mathrm{~K}$ line than in the Balmer lines. This shows that the chromospheric emission lines can also be affected by small flares, when the continuum exhibits no changes. Moreover, the chromospheric emission lines exhibit variability that is not seen in the X-ray band, thus should be confined to the chromosphere; for instance, in night 2, during the first two hours, the $\mathrm{H} \alpha$ and other chromospheric emission lines display a flux decline, but the X-ray level is constant, and in night 1 , the chromospheric lines show a peak at about 7:00 UT that is not noticed in the X-ray or continuum. This behaviour during flares has been found before. Osten et al. (2005b) found at radio, optical, and X-ray wavelengths for the flare star EV Lac flares with no counterparts at other wavelengths. In addition Hilton et al. (2010) searched for flares in the SDSS and found 243 flaring spectra of 63 flare stars with only two having an enhanced continuum. Continuum enhancement occurs especially for the strongest flares (see e.g. Kowalski et al. 2010a; and Hawley \& Fisher 1992).

We also analysed Proxima Centauri's integrated flux using PN spectra from the three individual XMM-Newton observations. The X-ray flux levels did not change significantly for the first and second exposure, whereas there is a significant change in the $\mathrm{X}$ ray flux in the third exposure as can be seen in Fig. 4; here the $\mathrm{X}$-ray flux was determined using the Xspec best-fit spectral models (see Sect. 4) integrated in the energy range 0.2 to $10.0 \mathrm{keV}$. Specifically, the night 1 EPIC PN exposure results in an Xray flux of $3.0 \times 10^{-12} \mathrm{erg} \mathrm{cm}^{-2} \mathrm{~s}^{-1}\left(L_{\mathrm{x}}=6.0 \times 10^{26} \mathrm{erg} \mathrm{s}^{-1}\right)$, the exposure of night 2 corresponds to $3.2 \times 10^{-12} \mathrm{erg} \mathrm{cm}^{-2} \mathrm{~s}^{-1}$ $\left(L_{\mathrm{x}}=6.4 \times 10^{26} \mathrm{erg} \mathrm{s}^{-1}\right)$, and the data from night 3 give 


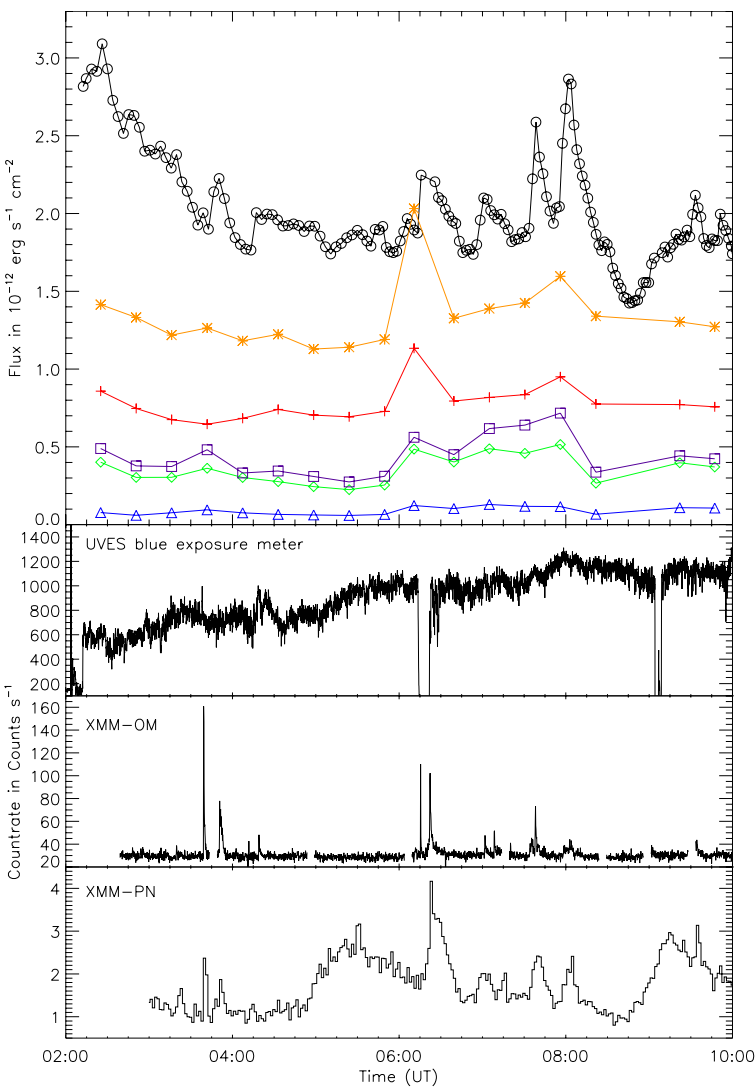

Fig. 2. As Fig. 1, but for night 2. The two gaps in the UVES light curve are due to technical problems that led to the loss of the guide star. The legend is the same as in Fig. 1.

$6.3 \times 10^{-12} \mathrm{erg} \mathrm{cm}^{-2} \mathrm{~s}^{-1}\left(L_{\mathrm{x}}=1.2 \times 10^{27} \mathrm{erg} \mathrm{s}^{-1}\right)$. The higher value for the third night is caused by the strong flare.

During all three nights, the X-ray flux in the quiescent time intervals is at a level of $2.6 \times 10^{-12} \mathrm{erg} \mathrm{cm}^{-2} \mathrm{~s}^{-1}\left(L_{\mathrm{x}}=4.9 \times\right.$ $10^{26} \mathrm{erg} \mathrm{s}^{-1}$ ). This is comparable to the lower boundary of the variable quiescent X-ray luminosity $4-16 \times 10^{26} \mathrm{erg} \mathrm{s}^{-1}$ found by Haisch et al. (1990).

We can calculate the activity level of Proxima Centauri using the activity indicator $\log L_{\mathrm{X}} / L_{\text {bol }}$. With infrared $H$ and $K$ band magnitudes of $m_{H}=4.835$ and $m_{K}=4.384$ and using the bolometric corrections given by Reid et al. (2001), Proxima Centauri's bolometric luminosity is $6 \times 10^{30} \mathrm{erg} \mathrm{s}^{-1}$, i.e., slightly less than the $L_{\text {bol }}=6.7 \times 10^{30} \mathrm{erg} \mathrm{s}^{-1}$ found by Frogel et al. (1972). With an activity indicator of $\log L_{X} / L_{\text {bol }}=-4$ in nonflaring time intervals, Proxima Centauri is a moderately active star.

\subsection{The large flare}

The X-ray light curve from night 3 (Fig. 3) shows a large flare with a peak X-ray flux of $3.8 \times 10^{-11} \mathrm{erg} \mathrm{cm}^{-2} \mathrm{~s}^{-1}\left(L_{\mathrm{x}}=\right.$ $7.2 \times 10^{27} \mathrm{erg} \mathrm{s}^{-1}$ ), which is also evident in the OM and optical data. The optical UVES exposure-meter and OM light curves resemble those of a typical solar impulsive flare. The X-ray data and light curves of chromospheric emission lines show a more complex behaviour with two broad secondary events peaking at about 6:45 and 9:10 UT in the X-ray light curve. In the $\mathrm{H} \alpha$ emission, we find an even more complicated behaviour with several sub-peaks in the decay phase, which are not seen in any of the other light curves but are roughly associated with the two

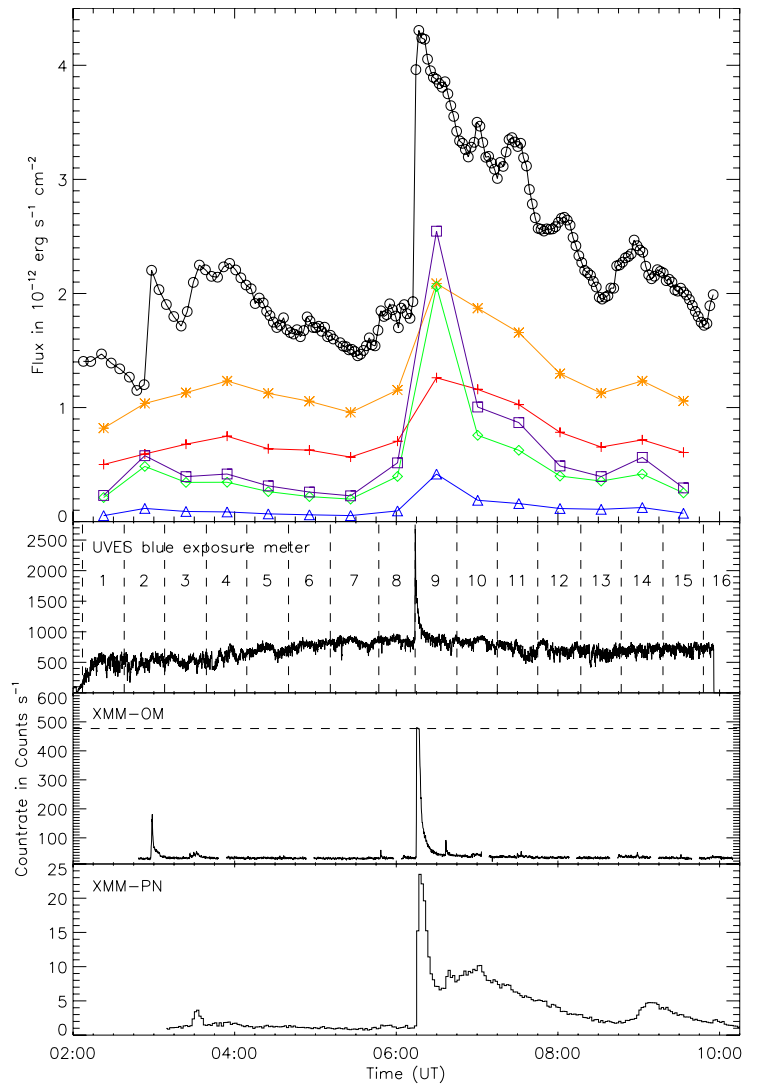

Fig. 3. As Fig. 1, but for night 3. The time intervals for the UVES blue arm spectra are also indicated for further reference. The dashed line in the OM panel marks the saturation limit that was reached in the flare peak. The figure legend is the same as in Fig. 1.

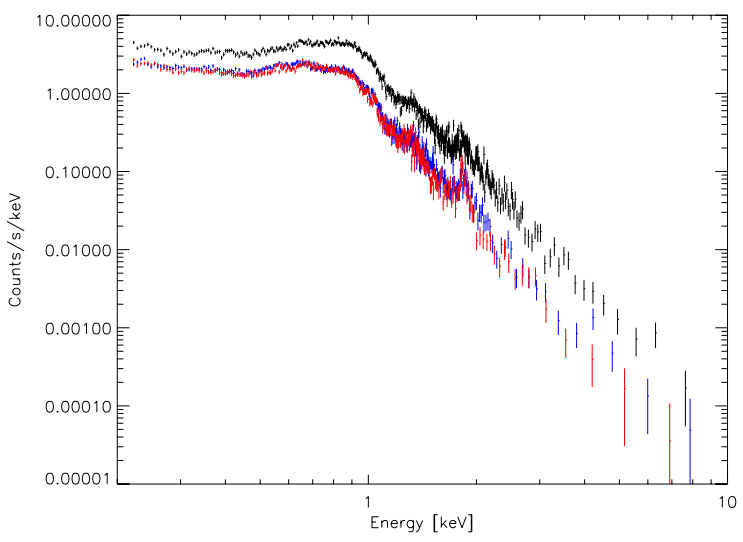

Fig. 4. PN spectra from night 1 (bottom/red), night 2 (middle/blue), and night 3 (top/black).

secondary peaks seen in X-rays. The two secondary peaks can also be identified in the other Balmer lines and the $\mathrm{Ca}$ II $\mathrm{H}$ and $\mathrm{K}$ lines despite their lower time resolution. The secondary peaks are found in neither the optical UVES light curve, nor the OM light curve. A similar event was described for Proxima Centauri by Güdel et al. (2002).

The flare rise to the maximum flux took place on a timescale of $\sim 500 \mathrm{~s}$. With a $10 \mathrm{~s}$ binning for X-ray and optical data, we found that the optical peak as seen in the OM light curve precedes the X-ray peak by about two minutes. This can be explained in terms of the Neupert effect (Neupert 1968) known 


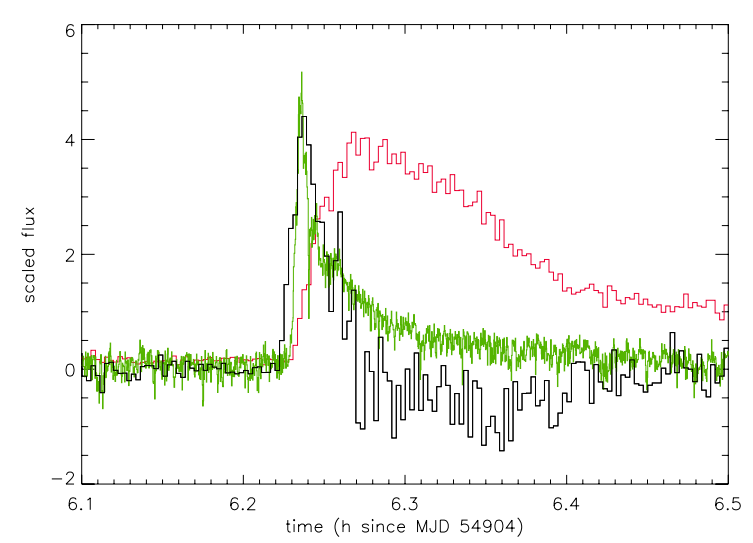

Fig. 5. Illustration of the Neupert effect during the large flare of Proxima Centauri on night 3. Depicted are the combined EPIC X-ray light curve in red, its time derivative (smoothed by five bins) in black, and the optical UVES light curve in green.

to occur in solar and a variety of stellar flares, where the time integral of emission due to particle acceleration, such as radio emission or blue continuum emission, resembles the rise of the flare light curve in soft X-rays. The explanation of this behaviour is that accelerated particles hit the dense chromosphere and that chromospheric material is heated and evaporates into the corona, where it accumulates and provides the emission measure of the subsequent soft X-ray emission.

This effect is also clearly seen in our data, because the time derivative of the combined EPIC (MOS1, MOS2 and PN) X-ray light curve during the flare rise (see Fig. 5) matches the shape of the optical light curve; we note that we cannot use the OM light curve for this analysis, since the OM data are saturated during the flare peak, but we use the light curve that is extracted from the UVES's blue-arm photometer.

\subsection{Average magnetic field}

The average magnetic field $B f$ of Proxima Centauri is measured using our UVES red arm data by employing a method introduced by Reiners \& Basri (2006) utilising an absorption band of molecular $\mathrm{FeH}$ close to $1 \mu \mathrm{m}$. We provide a brief overview of the method here and refer to the paper by Reiners \& Basri (2006) for a more detailed description of the procedure. The $\mathrm{FeH}$ band contains a large number of isolated lines, some of which are highly sensitive to the Zeeman effect, while others are not. Since Landé- $g$ factors are unavailable, a direct determination of the Zeeman effect is difficult, although there have been some attempts to model FeH molecular lines theoretically (see Shulyak et al. 2010). We measure the magnetic field by comparing observed $\mathrm{FeH}$ lines to spectra of stars with known magnetic fields. These template stars are GJ 1002 (M5.5, no measured magnetic field) and Gl 873 (M3.5, Bf 3.9 kG, Johns-Krull \& Valenti 2000). Before the spectra can be compared, the template spectra have to be scaled to match the strengths of the FeH absorption to the strength found in our spectra of Proxima Centauri. They also have to be adjusted to match in terms of rotational velocity. The observed spectrum is then modelled as a linear combination of the template spectra to determine $B f$, thus it is assumed that the Zeeman broadening is linear in $B f$ and that the magnetic field strength is distributed similarly in all stars.

We measured the average magnetic field $B f$ for all three nights by using a $\chi^{2}$-minimisation to find the best-fit relation for

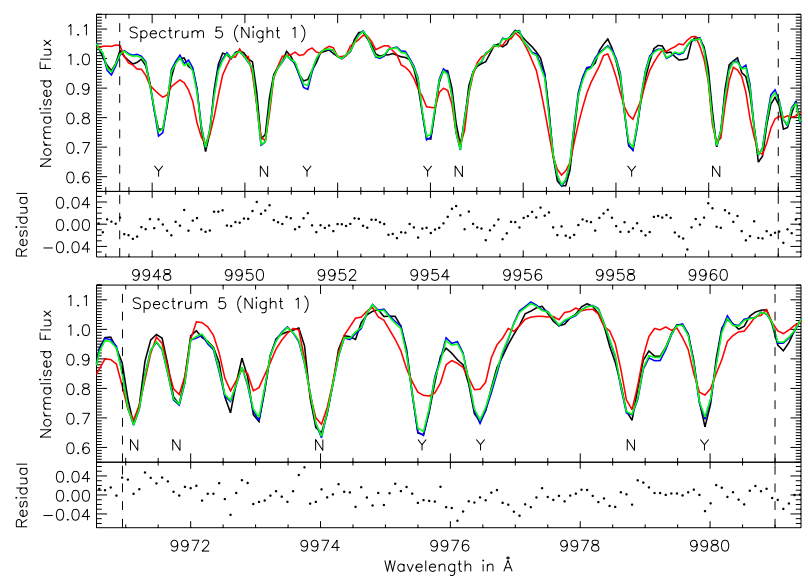

Fig. 6. Result of the fit for UVES red arm spectrum 5 from night 1 with $B f=400 \mathrm{G} \pm 110 \mathrm{G}$. Black: slope corrected Proxima Centauri data; red: scaled and broadened spectrum of magnetic reference G1 873; blue: scaled and broadened spectrum of non-magnetic reference GJ 1002; green: best fit; $Y$ : magnetically sensitive lines; $N$ : magnetically insensitive lines; vertical dashed lines border the wavelength regions used for the fit.

the interpolation between the template stars and the UVES red arm spectra of Proxima Centauri. The fit was calculated in two wavelength regions, 9947.3-9961.5 $\mathrm{A}$ and 9970.95-9981.0 ̊, containing 14 absorption lines of $\mathrm{FeH}$ with 7 being sensitive and 7 being insensitive to the magnetic field, respectively. A typical example of our fits is shown in Fig. 6. We compute a formal $1 \sigma$ uncertainty in $B f$ by searching the range of $B f$ for which $\chi^{2}<\chi_{\min }^{2}+1$ while varying the four other parameters. This results in an average uncertainty of $\overline{\Delta B f}=110 \mathrm{G}$. We note that it is only the statistical error that we use to compare individual differential measurements because the magnetically insensitive lines remain constant; thus they provide an accurate calibration particularly when intercomparing exposures. In addition to statistical errors, there are systematic errors and we estimate the true uncertainty in the absolute measurement of the magnetic field $B f$ to be of the order of a few hundred gauss. Hence, to accurately assess any trend in the data the statistical error has to be considered, while the systematic errors may offset the whole graph.

There is a relatively strong correlation between the seeing conditions and the derived values of $B f$, especially in the second and third night with Pearson's product-moment correlation coefficients $r$ of $r_{\mathrm{N} 2}=0.84$ and $r_{\mathrm{N} 3}=0.64$. This was eliminated by dividing the $B f$ measurements by a polynomial describing the general trend of the seeing taken from the ESO archive. The resulting timing behaviour of $B f$ is shown in Fig. 7. The field has a mean level of $\overline{B f}=250 \mathrm{G}$ with a statistical error of $60 \mathrm{G}$. Reiners \& Basri (2008) also measured the average magnetic field of Proxima Centauri with the same method using data taken in April 2004 and found magnetic field values in the range $450 \mathrm{G}$ $<B f<750 \mathrm{G}$. This change of $\sim 350 \mathrm{G}$ over five years might well be real, but is close to the limits of our uncertainty. Hence, it is unclear whether this difference is real or a consequence of the measuring inaccuracy.

As mentioned above, we can use the statistical error in the $\chi^{2}$-minimisation to compare individual measurements, because we are only interested in a differential comparison and not in the absolute values of $B f$. For the first and second nights, the magnetic field is consistent with a constant value within the error bars. For the third night, there is a dip in the magnetic field 


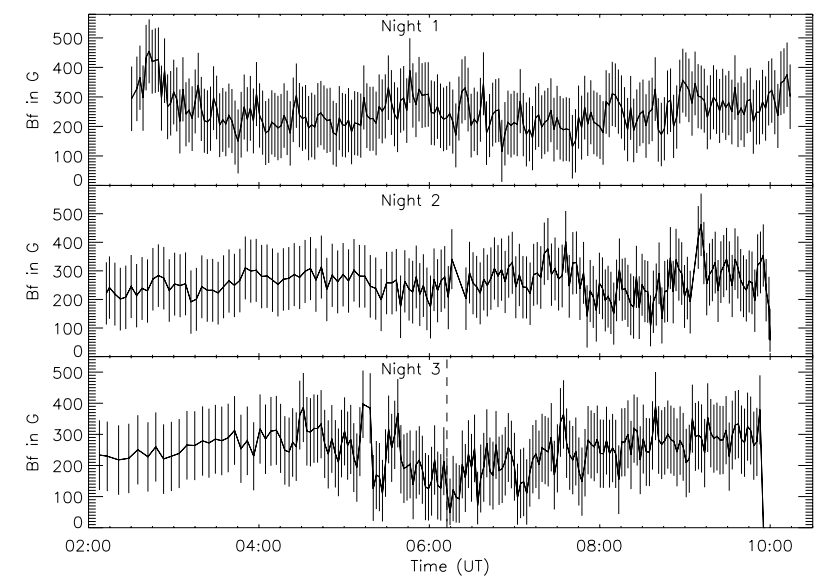

Fig. 7. Timing behaviour of the average magnetic field $B f$ for the three observed nights. The onset of the flare in the third night is marked by a vertical dashed line.

with its minimum roughly at the time of the flare onset (Fig. 7). In the context of the reconnection model for flares, a weak magnetic field at flare onset is what one would expect. However, we note that the measured fields refer to the photosphere, while the field annihilation is assumed to take place in the corona. In addition, the slow decay before the flare event is surprising for a catastrophic event such as magnetic reconnection, while after the flare the magnetic field recovers relatively quickly. In summary, it remains unclear whether there is a physical association of the magnetic field changes with the flare.

\section{Coronal properties of Proxima Centauri}

\subsection{X-ray spectral analysis}

Our X-ray observations of Proxima Centauri can be analysed to help us derive several time-variable properties of its corona such as temperatures, abundances, and densities, as we investigate in this section.

\subsubsection{Spectral fits and elemental abundances}

To visualise the spectral changes of Proxima Centauri associated with the large flare, we show the time evolution of the RGS spectra during night 3 in Fig. 8. The mean spectrum is shown in the uppermost part of the diagram, while the time evolution in the form of the light curve is depicted vertically at the right border. The mean spectrum resembles a typical RGS spectrum of M-dwarfs with $\mathrm{O}$ VIII Ly $\alpha$ being the strongest line and other prominent lines originating from Ne Ix, Ne x, Fe xvII, O vII, and $\mathrm{C}$ vi. As expected, the diagram shows a brightening in the spectral lines with the flare onset. The higher plasma temperatures during the flare manifest themselves as a relative brightening of lines formed at higher coronal temperatures than the cooler spectral lines, for example $\mathrm{O}$ vIII vs. the $\mathrm{O}$ vII triplet. However, pronounced changes in the density-sensitive ratio of the $\mathrm{O}$ VII forbidden to intercombination line cannot be identified in this time-resolved plot for the flare and the pre-flare period; they are only evident in time-integrated measurements for the flaring and quiescent states (see Sect. 4.1.3).

There is particular interest in studying elemental abundances during a flare. Since flares transport chromospheric material into the corona by chromospheric evaporation, coronal abundance patterns can temporarily change during flares. We determined the temperatures and abundances relative to solar values (Grevesse \& Sauval 1998) with an iterative procedure of global Xspec fits to EPIC and RGS spectra with VAPEC plasma models. In these fits, the abundances and emission measure are inherently interdependent, hence we made our inferences from relative changes in the fit parameters for the three observations. The errors we give for the fitted parameters were calculated with Xspec's error command. The program Xspec calculates the confidence intervals for the desired parameter by fixing this parameter at a specific value and varying the other free parameters until the best fit is reached. New fixed values for the parameter of interest are chosen until the error margins that were requested in the error command are covered. The errors in these plasma fits are correlated; in particular for the emission measure and elemental abundances, there is a strong interdependence (see below). Thus, in the calculation of Xspec's error margins for an individual parameter, all other non-frozen parameters are also allowed to vary, yielding effectively larger error margins than if one (incorrectly) assumes the errors to be uncorrelated.

When fitting the EPIC spectra, we adopted the following method to obtain the abundances: the abundances of oxygen, neon, iron, magnesium, and silicon were allowed to vary freely and independently, but were constrained to be the same among all the VAPEC temperature components. The abundances of all other elements were frozen to the solar value because they cannot be well-constrained with EPIC spectra since they do not have prominent spectral lines at energies where EPIC has a large effective area. When fitting EPIC spectra together with RGS spectra, carbon and nitrogen abundances also were allowed to vary. To determine the number of VAPEC components with different temperatures, we added new components one by one until the addition of the next component did not improve the fit significantly.

In the following sections we used the RGS spectra with their much higher wavelength resolution for spectral fitting simultaneously to the MOS spectra. We combined the spectra from the quiescent states of all three nights (for a definition of quiescence, see Sect. 3.1) and compared them to the spectra obtained from the large flare during the third night. To compare these two states, we defined a fixed temperature grid consisting of the values $0.14,0.4$, and $1.0 \mathrm{keV}(1.6,4.6$, and $11.5 \mathrm{MK})$. The resulting best-fit $\chi^{2}$ values of the three-temperature VAPEC models along with average individual elemental abundances for the quiescent state and the flaring state are listed in Table 2. The quiescent state is characterised by dominant plasma components at 0.14 and $0.4 \mathrm{keV}$. During the flare, there was a pronounced enhancement of the emission measure at 0.4 and $1.0 \mathrm{keV}$. The emission measure at low temperatures is more weakly constrained than for the quiescent state; within the errors, the low-temperature emission measure is consistent in both fits.

\subsubsection{The FIP effect}

Similar to the Sun, inactive or low-activity-level stars show a normal first ionisation potential (FIP) effect where elements with a low FIP are enhanced in the corona compared to elements with a high FIP. A reversed pattern - the inverse FIP effect - with enhanced high-FIP elements and depleted low-FIP elements is frequently found in stars of higher activity (e.g. Brinkman et al. 2001, Audard et al. 2003). A FIP effect is common among stars with low activity levels $\left(\log L_{\mathrm{X}} / L_{\mathrm{bol}}<-4\right)$. Owing to the activity level shown by Proxima Centauri, one would expect to observe the inverse FIP-effect. In the measurements for the quiescent state, the inverse FIP-effect might be observed but owing 


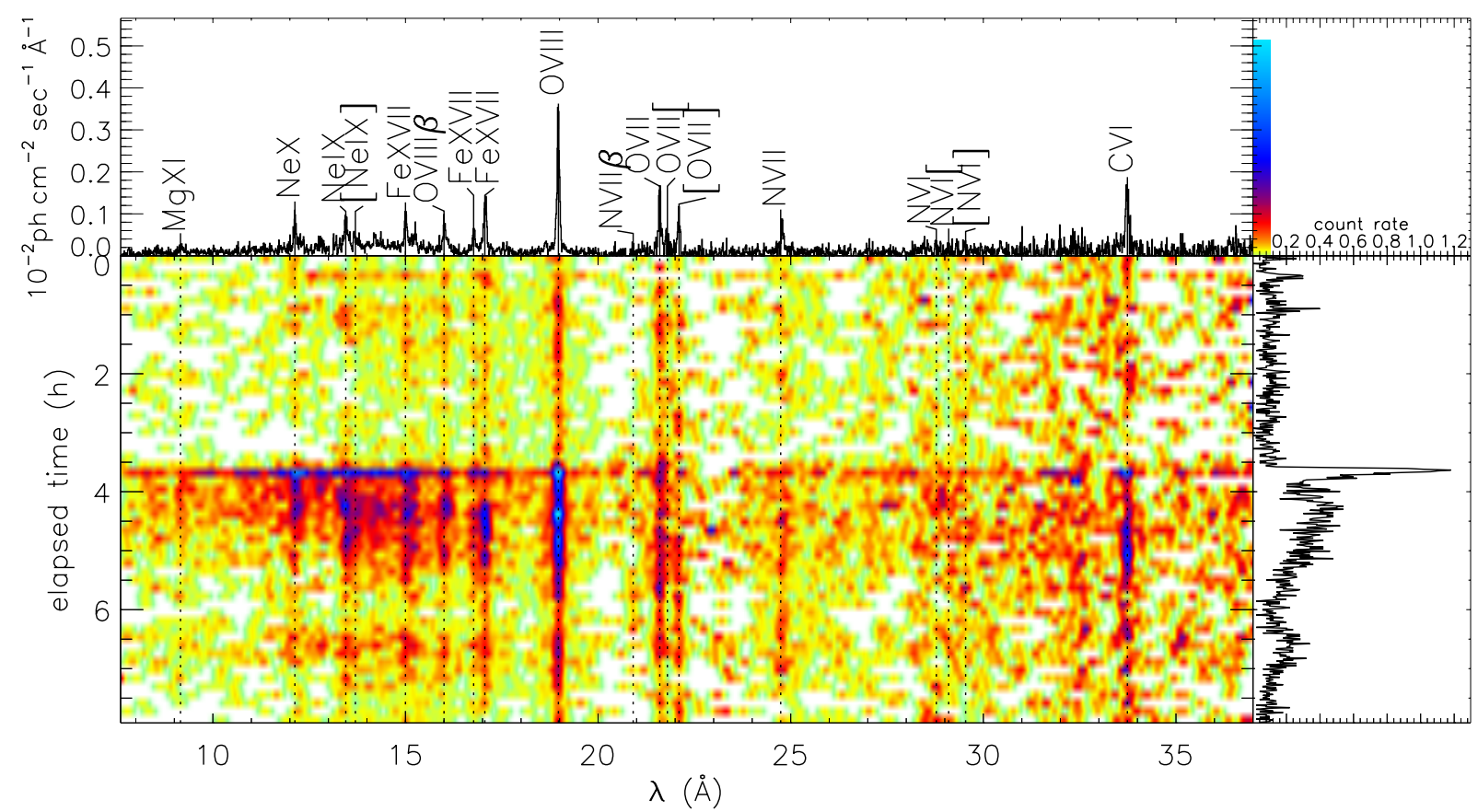

Fig. 8. Time evolution of RGS spectra of Proxima Centauri. Prominent emission lines are labelled in the mean spectrum shown in the upper part of the graph. In the right panel, the associated light curve can be found.

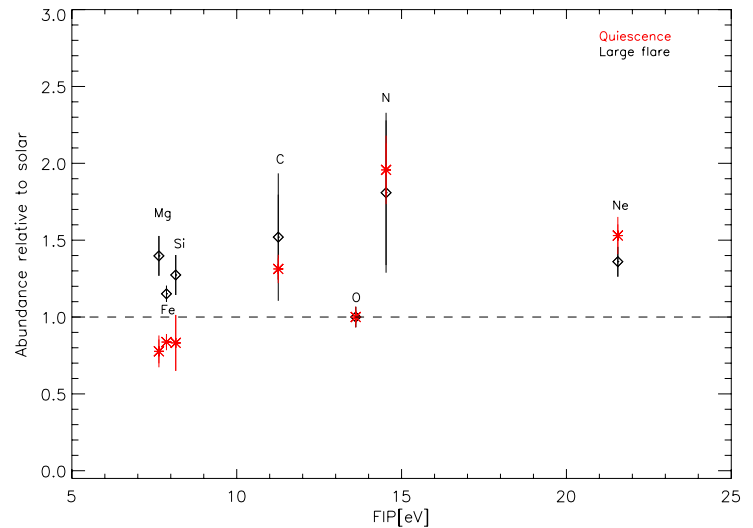

Fig. 9. Normalised elemental abundances to oxygen abundance relative to solar photospheric values (Grevesse \& Sauval 1998) as a function of the first ionisation potential (FIP) during quiescence (red) and flare (black). Dashed line indicates the solar photospheric abundance.

to our measurement errors we cannot make a strong claim here, which is illustrated in Fig. 9. The actual abundance values can be found in Table 2. During the flare, the abundances are slightly higher on average, and the iron and silicon abundances rise in relation to the high-FIP elements. This is in line with the picture that fresh chromospheric material is evaporated into the corona during the flare, which causes a change in elemental abundances. In previous X-ray observations of Proxima Centauri, a similar abundance distribution was found (Güdel et al. 2004).

To investigate in greater detail the abundance variations, we created spectra by dividing the flare data into several time intervals with the first four lasting $120 \mathrm{~s}$, followed by three intervals of $180 \mathrm{~s}, 240 \mathrm{~s}$, and $300 \mathrm{~s}$ and the remaining data being divided into spectral intervals of $600 \mathrm{~s}$ each. The first spectrum covers the flare rise and the following time intervals cover the different phases of the decay. These spectra are fitted with
Table 2. Three-temperature fit to the X-ray spectra extracted from MOS1, MOS2, RGS1, and RGS2 data allowing individual elemental abundance and emission measure to vary on a fixed temperature grid. Errors given are $1 \sigma$ errors.

\begin{tabular}{lcc}
\hline \hline Parameters & Quiescence & Large flare \\
\hline$T_{1}(\mathrm{keV})$ & \multicolumn{2}{c}{0.14} \\
$E M_{1}\left(10^{50} \mathrm{~cm}^{-3}\right)$ & $0.16_{-0.02}^{+0.02}$ & $0.17_{-0.07}^{+0.07}$ \\
$T_{2}(\mathrm{keV})$ & \multicolumn{2}{c}{0.4} \\
$E M_{2}\left(10^{50} \mathrm{~cm}^{-3}\right)$ & $0.35_{-0.02}^{+0.02}$ & 1.0 \\
$T_{3}(\mathrm{keV})$ & \multicolumn{2}{c}{$1.02_{-0.07}^{+0.08}$} \\
$E M_{3}\left(10^{50} \mathrm{~cm}^{-3}\right)$ & $0.01_{-0.01}^{+0.01}$ & $1.10_{-0.03}^{+0.03}$ \\
$\mathrm{C}$ & $0.57_{-0.03}^{+0.04}$ & $0.66_{-0.12}^{+0.18}$ \\
$\mathrm{~N}$ & $0.79_{-0.07}^{+0.09}$ & $0.73_{-0.19}^{+0.21}$ \\
$\mathrm{O}$ & $0.45_{-0.02}^{+0.03}$ & $0.45_{-0.03}^{+0.03}$ \\
$\mathrm{Ne}$ & $0.63_{-0.03}^{+0.05}$ & $0.56_{-0.04}^{+0.04}$ \\
$\mathrm{Mg}$ & $0.30_{-0.03}^{+0.04}$ & $0.54_{-0.05}^{+0.05}$ \\
$\mathrm{Si}$ & $0.32_{-0.07}^{+0.07}$ & $0.49_{-0.05}^{+0.05}$ \\
$\mathrm{Fe}$ & $0.32_{-0.02}^{+0.02}$ & $0.44_{-0.02}^{+0.02}$ \\
red. $\chi^{2}$ & 1.96 & 1.877 \\
$\mathrm{D} . \mathrm{O} . \mathrm{F}$. & 784 & 561 \\
$\log L_{\mathrm{X}}(0.2-10 \mathrm{keV})$ & 26.70 & 27.50 \\
\hline
\end{tabular}

combinations of APEC models, using two temperature components and assuming fixed elemental abundances for the flare plasma but leaving the iron abundance as a free parameter. To obtain the plasma properties, we use the total emission measure $\mathrm{EM}$, i.e. the sum of the emission measures of each temperature component

$E M=\sum_{i} E M_{i}$ 
Table 3. Model parameters for the flare spectra with variable Fe abundances.

\begin{tabular}{|c|c|c|c|c|c|c|c|c|c|}
\hline $\begin{array}{l}\text { Spect. } \\
\text { No. }\end{array}$ & $\begin{array}{c}K T_{1} \\
{[\mathrm{keV}]}\end{array}$ & $\begin{array}{c}E M_{1} \\
{\left[10^{50} \mathrm{~cm}^{-3}\right]}\end{array}$ & $\begin{array}{c}K T_{2} \\
{[\mathrm{keV}]}\end{array}$ & $\begin{array}{c}E M_{2} \\
{\left[10^{50} \mathrm{~cm}^{-3}\right]}\end{array}$ & $\mathrm{Fe}$ & Red. $\chi^{2}$ & d.o.f & $\begin{array}{c}T \\
\mathrm{MK}\end{array}$ & $\begin{array}{c}E M \\
{\left[10^{50} \mathrm{~cm}^{-3}\right]}\end{array}$ \\
\hline 1 & $0.75_{-0.06}^{+0.05}$ & $2.38_{-0.63}^{+0.91}$ & $1.90_{-0.26}^{+0.28}$ & $4.90_{-0.72}^{+0.58}$ & $0.58_{-0.12}^{+0.15}$ & 1.15 & 119 & $17.71_{-1.95}^{+1.65}$ & $7.28_{-1.36}^{+1.49}$ \\
\hline 2 & $0.69_{-0.09}^{+0.07}$ & $3.23_{-0.85}^{+1.12}$ & $1.58_{-0.17}^{+0.19}$ & $6.39_{-0.98}^{+0.81}$ & $0.38_{-0.07}^{+0.12}$ & 1.10 & 148 & $14.88_{-1.61}^{+1.44}$ & $9.62_{-1.83}^{+1.93}$ \\
\hline 3 & $0.61_{-0.03}^{+0.03}$ & $2.85_{-0.44}^{+0.53}$ & $1.60_{-0.12}^{+0.11}$ & $4.98_{-0.42}^{+0.40}$ & $0.54_{-0.09}^{+0.11}$ & 1.19 & 159 & $14.40_{-0.91}^{+0.82}$ & $7.83_{-0.86}^{+0.94}$ \\
\hline 4 & $0.63_{-0.09}^{+0.03}$ & $3.61_{-1.87}^{+1.47}$ & $1.26_{-0.15}^{+0.27}$ & $2.85_{-0.92}^{+1.71}$ & $0.35_{-0.09}^{+0.11}$ & 0.95 & 93 & $10.60_{-1.32}^{+1.89}$ & $6.47_{-2.80}^{+3.19}$ \\
\hline 5 & $0.58_{-0.12}^{+0.07}$ & $2.41_{-0.54}^{+0.59}$ & $1.22_{-0.24}^{+0.48}$ & $1.91_{-0.62}^{+0.48}$ & $0.33_{-0.06}^{+0.07}$ & 1.07 & 113 & $10.05_{-2.17}^{+2.95}$ & $4.32_{-1.16}^{+1.07}$ \\
\hline 6 & $0.46_{-0.10}^{+0.13}$ & $1.80_{-0.36}^{+0.75}$ & $0.97_{-0.13}^{+0.14}$ & $1.71_{-0.75}^{+0.38}$ & $0.24_{-0.07}^{+0.11}$ & 0.92 & 96 & $8.26_{-1.41}^{+1.57}$ & $3.52_{-1.12}^{+1.13}$ \\
\hline 7 & $0.38_{-0.06}^{+0.09}$ & $1.16_{-0.18}^{+0.17}$ & $1.06_{-0.07}^{+0.11}$ & $1.67_{-0.11}^{+0.22}$ & $0.37_{-0.10}^{+0.20}$ & 1.20 & 106 & $9.11_{-0.75}^{+1.20}$ & $2.84_{-0.30}^{+0.40}$ \\
\hline 8 & $0.79_{-0.03}^{+0.02}$ & $2.72_{-0.46}^{+0.34}$ & $1.58_{-0.46}^{+0.89}$ & $0.79_{-0.39}^{+0.45}$ & $0.21_{-0.03}^{+0.05}$ & 1.00 & 135 & $11.28_{-2.69}^{+6.05}$ & $3.52_{-0.85}^{+0.79}$ \\
\hline 9 & $0.64_{-0.03}^{+0.02}$ & $1.71_{-0.44}^{+0.43}$ & $1.01_{-0.14}^{+0.12}$ & $1.82_{-0.35}^{+0.38}$ & $0.26_{-0.03}^{+0.04}$ & 1.03 & 229 & $9.64_{-0.97}^{+0.82}$ & $3.53_{-0.79}^{+0.82}$ \\
\hline 10 & $0.62_{-0.12}^{+0.02}$ & $2.86_{-0.33}^{+0.39}$ & $1.22_{-0.27}^{+0.42}$ & $0.92_{-0.32}^{+0.28}$ & $0.30_{-0.03}^{+0.03}$ & 1.03 & 222 & $8.88_{-2.32}^{+2.27}$ & $3.78_{-0.65}^{+0.67}$ \\
\hline 11 & $0.46_{-0.09}^{+0.03}$ & $1.88_{-0.69}^{+0.74}$ & $0.76_{-0.06}^{+0.13}$ & $2.00_{-0.87}^{+0.72}$ & $0.27_{-0.03}^{+0.05}$ & 1.00 & 217 & $7.17_{-0.91}^{+1.22}$ & $3.89_{-1.57}^{+1.47}$ \\
\hline 12 & $0.31_{-0.04}^{+0.03}$ & $1.52_{-0.32}^{+0.29}$ & $0.72_{-0.04}^{+0.05}$ & $1.72_{-0.29}^{+0.34}$ & $0.36_{-0.06}^{+0.07}$ & 0.92 & 193 & $6.21_{-0.51}^{+0.52}$ & $3.24_{-0.62}^{+0.64}$ \\
\hline 13 & $0.24_{-0.03}^{+0.05}$ & $1.25_{-0.34}^{+0.45}$ & $0.64_{-0.03}^{+0.05}$ & $1.70_{-0.37}^{+0.35}$ & $0.38_{-0.05}^{+0.07}$ & 0.81 & 184 & $5.53_{-0.43}^{+0.62}$ & $2.95_{-0.72}^{+0.80}$ \\
\hline 14 & $0.25_{-0.04}^{+0.05}$ & $1.04_{-0.35}^{+0.50}$ & $0.59_{-0.03}^{+0.06}$ & $1.42_{-0.46}^{+0.38}$ & $0.38_{-0.07}^{+0.09}$ & 0.97 & 162 & $5.23_{-0.47}^{+0.73}$ & $2.47_{-0.82}^{+0.89}$ \\
\hline 15 & $0.22_{-0.05}^{+0.03}$ & $0.88_{-0.40}^{+0.29}$ & $0.57_{-0.07}^{+0.04}$ & $1.36_{-0.29}^{+0.54}$ & $0.34_{-0.07}^{+0.08}$ & 1.14 & 142 & $5.05_{-0.74}^{+0.46}$ & $2.25_{-0.70}^{+0.83}$ \\
\hline 16 & $0.26_{-0.01}^{+0.03}$ & $1.67_{-0.12}^{+0.12}$ & $1.78_{-0.09}^{+0.15}$ & $0.40_{-0.14}^{+0.20}$ & $0.35_{-0.17}^{+0.21}$ & 0.75 & 134 & $4.28_{-0.69}^{+1.30}$ & $2.08_{-0.27}^{+0.32}$ \\
\hline 17 & $0.24_{-0.01}^{+0.01}$ & $1.31_{-0.11}^{+0.10}$ & $0.78_{-0.28}^{+0.19}$ & $0.25_{-0.09}^{+0.44}$ & $0.37_{-0.03}^{+0.05}$ & 1.11 & 103 & $3.82_{-1.61}^{+1.83}$ & $1.57_{-0.21}^{+0.55}$ \\
\hline 18 & $0.24_{-0.02}^{+0.02}$ & $1.15_{-0.09}^{+0.14}$ & $0.70_{-0.09}^{+0.13}$ & $0.25_{-0.14}^{+0.11}$ & $0.36_{-0.17}^{+0.37}$ & 1.02 & 91 & $3.78_{-0.78}^{+0.83}$ & $1.40_{-0.24}^{+0.25}$ \\
\hline 19 & $0.31_{-0.04}^{+0.09}$ & $1.15_{-0.30}^{+0.29}$ & $0.68_{-0.22}^{+0.35}$ & $0.21_{-0.19}^{+0.26}$ & $0.29_{-0.07}^{+0.07}$ & 0.95 & 74 & $4.29_{-1.39}^{+2.54}$ & $1.37_{-0.50}^{+0.56}$ \\
\hline 20 & $0.22_{-0.02}^{+0.02}$ & $0.79_{-0.09}^{+0.08}$ & $0.69_{-0.09}^{+0.14}$ & $0.15_{-0.06}^{+0.09}$ & $0.20_{-0.11}^{+0.07}$ & 1.24 & 62 & $3.48_{-0.61}^{+1.01}$ & $0.95_{-0.16}^{+0.18}$ \\
\hline 21 & $0.13_{-0.01}^{+0.03}$ & $0.72_{-0.19}^{+0.23}$ & $0.47_{-0.06}^{+0.14}$ & $0.54_{-0.30}^{+0.19}$ & $0.24_{-0.08}^{+0.05}$ & 0.98 & 60 & $3.25_{-0.54}^{+0.97}$ & $1.26_{-0.50}^{+0.43}$ \\
\hline 22 & $0.23_{-0.03}^{+0.02}$ & $0.70_{-0.13}^{+0.19}$ & $0.76_{-0.10}^{+0.10}$ & $0.45_{-0.13}^{+0.15}$ & $0.32_{-0.12}^{+0.16}$ & 1.03 & 77 & $5.14_{-0.84}^{+0.73}$ & $1.15_{-0.26}^{+0.34}$ \\
\hline 23 & $0.40_{-0.06}^{+0.08}$ & $1.05_{-0.13}^{+0.21}$ & $0.91_{-0.11}^{+0.07}$ & $1.00_{-0.17}^{+0.25}$ & $0.33_{-0.05}^{+0.08}$ & 1.09 & 135 & $7.57_{-1.05}^{+0.94}$ & $2.05_{-0.31}^{+0.46}$ \\
\hline 24 & $0.26_{-0.04}^{+0.07}$ & $0.76_{-0.23}^{+0.31}$ & $0.72_{-0.05}^{+0.08}$ & $1.22_{-0.28}^{+0.25}$ & $0.27_{-0.06}^{+0.08}$ & 0.84 & 129 & $6.38_{-0.59}^{+0.90}$ & $1.98_{-0.52}^{+0.57}$ \\
\hline 25 & $0.19_{-0.03}^{+0.03}$ & $0.66_{-0.14}^{+0.13}$ & $0.63_{-0.04}^{+0.04}$ & $0.90_{-0.17}^{+0.19}$ & $0.32_{-0.07}^{+0.09}$ & 1.14 & 105 & $5.16_{-0.44}^{+0.48}$ & $1.56_{-0.31}^{+0.32}$ \\
\hline 26 & $0.29_{-0.05}^{+0.06}$ & $0.94_{-0.22}^{+0.22}$ & $0.78_{-0.14}^{+0.11}$ & $0.38_{-0.11}^{+0.21}$ & $0.32_{-0.15}^{+0.28}$ & 1.22 & 85 & $5.09_{-1.00}^{+1.02}$ & $1.33_{-0.34}^{+0.44}$ \\
\hline 27 & $0.26_{-0.03}^{+0.06}$ & $0.72_{-0.18}^{+0.17}$ & $0.68_{-0.10}^{+0.12}$ & $0.32_{-0.10}^{+0.18}$ & $0.32_{-0.11}^{+0.18}$ & 1.02 & 69 & $4.56_{-0.72}^{+1.09}$ & $1.04_{-0.29}^{+0.36}$ \\
\hline 28 & $0.23_{-0.11}^{+0.02}$ & $0.65_{-0.16}^{+0.09}$ & $0.78_{-0.16}^{+0.11}$ & $0.25_{-0.08}^{+0.21}$ & $0.25_{-0.20}^{+0.11}$ & 1.14 & 66 & $4.49_{-1.53}^{+1.03}$ & $0.91_{-0.25}^{+0.31}$ \\
\hline
\end{tabular}

and the flare temperature $T$ is defined as an emission-measure weighted sum of the temperatures from each flare component

$T=\sum_{i} \frac{T_{i} \times E M_{i}}{E M}$.

We infer that the iron abundance increases from a level of $0.30 \pm 0.03$ directly before the onset of the flare to a maximum value of $0.58_{-0.12}^{+0.15}$, when the flare peak is reached (see Table 3 ). As oxygen is another element that produces very strong emission lines in our X-ray spectra, we also investigated possible oxygen abundance changes during the flare in the same fashion, but found no evidence of a similar timing behaviour. This indicates that fresh plasma material is evaporated from the photosphere and chromosphere that has a different composition with a higher iron abundance than the quiescent coronal plasma. This scenario fits very well with the measured $\mathrm{H} \alpha$ asymmetries that indicate movements from the chromosphere in the direction of the corona (see Sect. 5.2). As can be seen from line shift measurements in Table 9, material is evaporated with a velocity of $44.3 \mathrm{~km} \mathrm{~s}^{-1}$ in the spectrum lasting from 6:12 to 6:14 UT. If one makes a conservative estimate that the material is accelerated only at the end of the exposure time, it can travel for about 90 seconds or about $4000 \mathrm{~km}$ before the measurement No. 1 of Table 3 starts (6:16:00 UT), which would place the material within the corona. For the second measurement of $\mathrm{H} \alpha$ blue-shifts, the situation is not as clear because the blue-shifts persist for a longer time overlapping with measurement No. 3 (starting at 6:20:00 UT) of higher coronal iron abundance. If most of the material is evaporated at the beginning of the blue-shift, the material moves for about $210 \mathrm{~s}$ or about $2200 \mathrm{~km}$ before the X-ray measurement No. 3 starts, again putting the material into the corona. 
Table 4. X-ray counts in the $\mathrm{O}$ vir triplet during quiescence (total integration time of $49 \mathrm{ks}$ ) and during the flare (total integration time of $7 \mathrm{ks})$.

\begin{tabular}{lcc}
\hline \hline Line & Counts (quiescence) & Counts (flare) \\
\hline resonance $(21.6 \AA)$ & $225 \pm 17$ & $96 \pm 12$ \\
intercombination $(21.8 \AA)$ & $60 \pm 11$ & $39 \pm 8$ \\
forbidden $(22.1 \AA)$ & $130 \pm 13$ & $48 \pm 8$ \\
\hline
\end{tabular}

\subsubsection{Coronal densities}

Electron densities can be inferred from line triplets of He-like ions. The RGS energy range contains density-sensitive Helike triplets of $\mathrm{N}$ vi, $\mathrm{O}_{\text {viI, }} \mathrm{Ne}$ Ix, $\mathrm{Mg}$ XI, and $\mathrm{Si}$ XIII. These He-like triplets show in increasing order of wavelength a resonance $(r)$, intercombination $(i)$, and a forbidden line $(f)$. If the electron collision rate is sufficiently high, ions in the upper level of the forbidden transition, $1 \mathrm{~s} 2 \mathrm{~s}^{3} \mathrm{~S}_{1}$, do not return to the ground level, $1 \mathrm{~s}^{21} \mathrm{~S}_{0}$, but the ions are instead collisionally excited to the upper level of the intercombination transitions, $1 \mathrm{~s} 2 \mathrm{p}^{3} \mathrm{P}_{1,2}$, from where they decay radiatively to the ground state. Thus, the ratio of the $\mathrm{f}$ to $\mathrm{i}$ fluxes is sensitive to density (Gabriel \& Jordan 1969).

The He-like triplet of $\mathrm{O}_{\mathrm{VII}}$ is strong enough in our observations to be used to obtain characteristic electron densities in the source region. We estimate the coronal plasma densities using the density-sensitive ratio of the forbidden to intercombination line of the $\mathrm{O}$ VII triplet in the quiescent and the flaring state. The measured $f / i$ flux ratios are $2.17 \pm 0.61$ in quiescence and $1.23 \pm 0.46$ during the flare in the third night (see Fig. 10, Table 4). The errors in these line ratios are rather large and are caused by the weak intercombination line. Formally, the errors overlap, hence one could argue that the change in density is insignificant. However, the ratio $(f+i) / r$ is practically constant during quiescence $(0.84 \pm 0.17)$ and the flare $(0.90 \pm 0.28)$. This indicates that physically X-ray photons are only shifted from the forbidden to the intercombination line at high densities. Hence, the constant $(f+i) / r$ ratio suggests that the change in the $f / i$ ratio is actually density-related and makes it less probable that it is a statistical fluctuation.

To convert the measured $f / i$ ratios into coronal plasma densities, we approximated the flux ratio by $f / i=R_{\mathrm{o}} /\left(1+n_{\mathrm{e}} / N_{\mathrm{c}}\right)$, where $R_{\mathrm{o}}$ is the low density limit and $N_{\mathrm{c}}$ is the critical density. We adopted the values from Pradhan \& Shull (1981) of 3.95 and $3.1 \times 10^{10} \mathrm{~cm}^{-3}$, respectively. We find electron densities of $n_{\mathrm{e}}=$ $2.5 \pm 0.7 \times 10^{10} \mathrm{~cm}^{-3}$ in quiescence and $n_{\mathrm{e}}=6.9 \pm 2.6 \times 10^{10} \mathrm{~cm}^{-3}$ during the third-night flaring period. This suggests an increase in plasma density during the flare, as one expects from the standard flare model.

\subsection{Interpretation: loop properties}

Emission measure - temperature $(E M-T)$ diagrams are useful for estimating physical quantities that are not directly observable. Since there is no direct information about the morphology of the involved coronal structures, we estimate the size of the spatially unresolved stellar coronal flaring region from the light curve, time-resolved temperature $(T)$, and emission measure $(E M)$ values obtained during the decay of the flaring loop. The decay time of an X-ray flare is directly related to the length of the flaring loop. However, if there is a significant amount of heating present during the decay of the flare, it can prolong the decay and lead to an overestimated loop length. We assumed
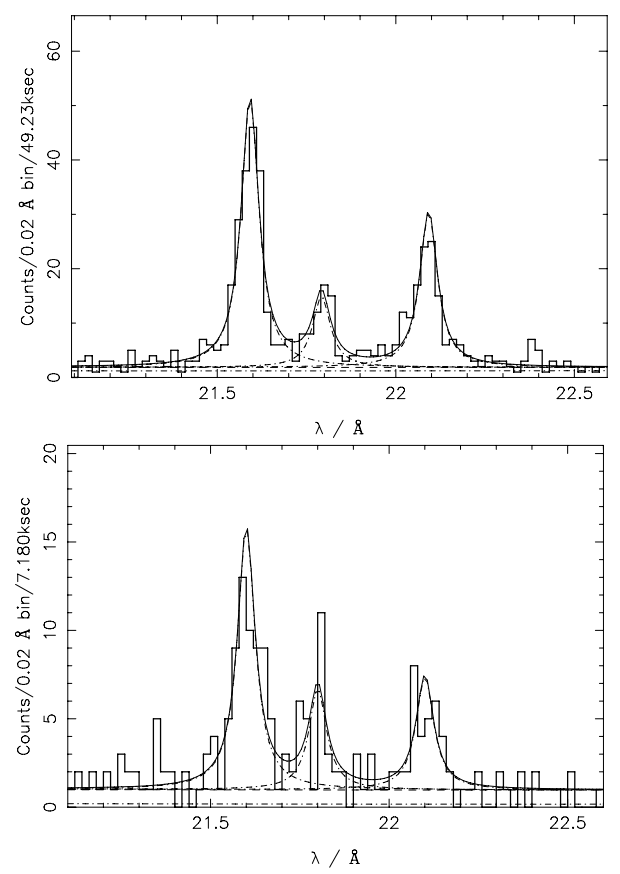

Fig. 10. Density-sensitive line triplet of O VII (resonance, intercombination, and forbidden line for increasing wavelength). The upper panel shows the best fit to cumulated RGS1 data during quiescence, while the lower panel shows the RGS1 triplet data during the large flare.

that the flare occurs inside a single coronal loop with a constant cross-section that is anchored in the photosphere. The plasma is confined to the loop, and the decay begins after the loop has reached a quasi-steady state, where the energy and plasma flow are negligible. According to this assumption, the decay time of the X-ray emission roughly scales with the plasma cooling time, which in turn scales with the length of the loop structure. Therefore, the longer the decay takes, the larger the loop structure becomes. Serio et al. (1991) derived a thermodynamic timescale for pure cooling of flaring plasma confined to a single flaring coronal loop. Reale et al. (1997) derived an empirical formula to determine the loop length considering the effect of sustained heating during the flare decay that uses the trajectory of the flare in the EM-T diagram, viz.

$L=\frac{\tau_{\mathrm{LC}} \sqrt{T_{0}}}{\alpha F(\zeta)}$ or $L_{9}=\frac{\tau_{\mathrm{LC}} \sqrt{T_{0,7}}}{120 F(\zeta)} \zeta_{\min }<\zeta \leq \zeta_{\max }$,

where $\tau_{\mathrm{LC}}$ is the decay time derived from the light curve and $\alpha=3.7 \times 10^{-4} \mathrm{~cm}^{-1} \mathrm{~s} \mathrm{~K}^{1 / 2}$. The observed maximum temperature must be corrected to $T_{0}(T-0,7)=\xi T_{\mathrm{obs}}^{\eta}$ (in units of $10^{7} \mathrm{~K}$ ), where $T_{\text {obs }}$ is the maximum best-fit temperature derived from spectral fitting to the data. The unit-less correction factor is $F(\zeta)=\frac{c_{a}}{\zeta-\zeta_{a}}+q_{a}$, where $\zeta$ is the slope of EM-T diagram. The coefficients $\xi, \eta, c_{a}, \zeta_{a}$, and $q_{a}$ depend on the energy response of the instrument. According to Reale (2007), for XMM/EPIC the values are $\xi=0.13, \eta=1.16, c_{a}=0.51, \zeta_{a}=0.35$, and $q_{a}=1.35$.

We show the $E M-T$ diagram of the flare on Proxima Centauri including the two secondary flares in Fig. 11. For the part of the flare evolution before the secondary events, the slope measured from the $\log T-0.5 \log$ EM diagram in Fig. 11 is $\zeta=1.07 \pm 0.29$. Given $T_{\text {peak }}[\mathrm{MK}]=33.26_{-11.93}^{+7.73}$, we determined the loop half length to be $L=8.55_{-2.86}^{+3.81} \times 10^{9} \mathrm{~cm}$. Assuming that the volume $V \propto L^{3}$, we computed the volume of the flaring region $V_{\text {flare }} \approx$ $6.25_{-0.35}^{+2.12} \times 10^{29} \mathrm{~cm}^{3}$. We also computed the loop-footpoint area 


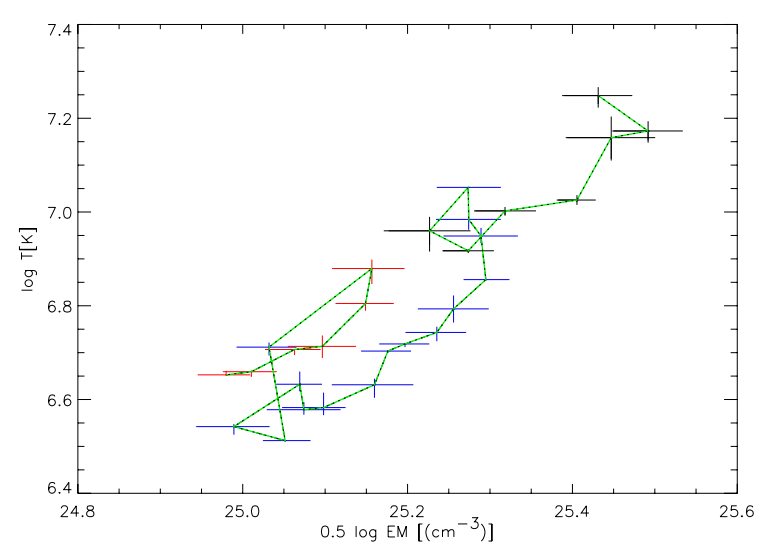

Fig. 11. Flare evolution in density-temperature. The green line represents the evolution of flare decay (black) and secondary events (blue and red).

$V / 2 L$ and an area filling-factor of the flare of three percent. We also note that in addition to this flare decay, there are two other secondary events. From the EM-T diagram for these events, the slopes measured from these secondary events are similar to the slope obtained for the first flare event. Thus, the derived loop lengths are of the same order of magnitude within the errors.

\subsection{Optical FexIII emission}

We also investigated the FexIII forbidden coronal line at 3388.1 $\AA$ in our spectra of Proxima Centauri. The stellar spectrum had previously been searched for this line but no clear conclusion had been drawn (Fuhrmeister et al. 2004). In the new UVES data, there is indeed evidence of the forbidden line during and after flare activity for the first and the third night.

To isolate the forbidden coronal line emission at $3388.1 \AA$, we averaged at least three normalised spectra for the quiescent state, the flare state, and after-flare state. To emphasise the chromospheric and coronal changes between the averaged spectra, we subtracted the quiescent state from the flaring state and the post-flare state (and for testing purposes also from different quiescent states of the same night). These difference spectra of the different quiescent states usually contain only noise as expected. However, the differences between the spectra of flare states and quiescent states in the first and third nights show a weak broad excess at the position of the Fe XIII line beginning at about UT 6:00 for the first night. This line persists for the post-flare state. In the third night, the line can also be found directly before the large flare starting at UT 4:40, while no evidence of Fe XIII emission could be found for the second night. An example of the line can be found in Fig. 12, the fit parameters obtained with CORA can be found in Table 5. The derived fluxes have an error of about a factor of two because of the uncertainties in the flux calibration. For the first night, we used the spectra No. 3-7 as quiescent state, for the second night the spectra No. 4-6, and for the third night the spectra No. 1-5. The half width $\sigma$ of the line is much larger than the typical measured half width of the chromospheric lines of $0.04 \AA$ (see Table 6 ). Interpreting the line width as pure thermal Doppler broadening, we expect a half width of $0.04 \AA$, for a typical chromospheric temperature of about $10000 \mathrm{~K}$ and a half width of about $0.17 \AA$ for the coronal Fe XIII line with a peak formation temperature of about 1.6 MK. The measured half widths are larger for the forbidden Fe XIII line suggesting

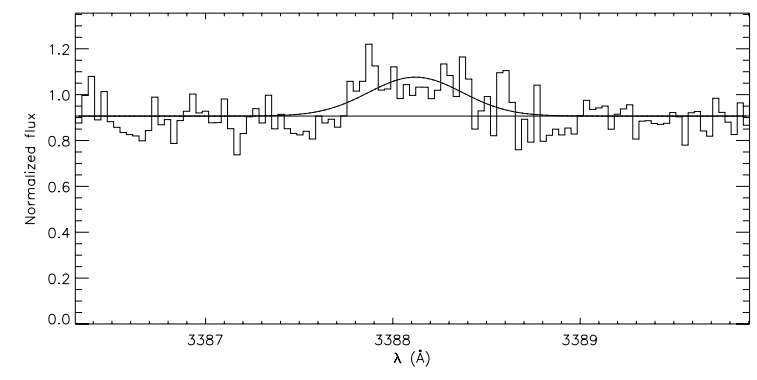

Fig. 12. Example of the fit to the averaged difference spectrum of the first night for the after-flare state at the end of the night for the Fe XIII line. The broad Gaussian is the line best fit obtained with CORA.

Table 5. Fit parameters of the Fe XIII line.

\begin{tabular}{lccc}
\hline \hline $\begin{array}{l}\text { Averaged } \\
\text { spectra No. }\end{array}$ & $\begin{array}{c}\text { Central wavelength } \\
{[\AA]}\end{array}$ & $\begin{array}{c}\text { Half width } \\
{[\AA]}\end{array}$ & $\begin{array}{c}\text { Line flux } \\
{\left[\mathrm{erg} \mathrm{s}^{-1} \mathrm{~cm}^{-2}\right]}\end{array}$ \\
\hline 1st night & & & \\
13-17 & $3388.02 \pm 1.32$ & $0.25 \pm 0.02$ & $6.6 \times 10^{-15}$ \\
18-22 & $3388.07 \pm 1.32$ & $0.22 \pm 0.02$ & $6.6 \times 10^{-15}$ \\
23-26 & $3388.10 \pm 1.32$ & $0.25 \pm 0.02$ & $7.2 \times 10^{-15}$ \\
\hline 3rd night & & & \\
6-8 & $3388.13 \pm 1.32$ & $0.27 \pm 0.03$ & $1.0 \times 10^{-14}$ \\
9-11 & $3388.16 \pm 1.32$ & $0.20 \pm 0.01$ & $1.5 \times 10^{-14}$ \\
12-15 & $3388.15 \pm 1.32$ & $0.36 \pm 0.03$ & $1.7 \times 10^{-14}$ \\
\hline
\end{tabular}

Notes. ${ }^{(a)}$ Flux errors are about a factor of two.

that there are additional line-broadening mechanisms; however, it must be kept in mind that the measurement of the line width is sensitive to the determination of the background, thus the given (formal) errors are probably underestimates.

The order of magnitude of the derived Fe XIII flux is consistent with our estimate derived from Proxima Centauri's coronal X-ray spectrum during the flare. The $\mathrm{O}$ vII triplet is formed at a similar temperature as Fe XIII $(\log T=6.3$ and 6.2, respectively). We calculate the flux in the $\mathrm{O}$ vII triplet during the flare from a CORA fit to the RGS1 spectrum to be $F_{\mathrm{OVII}}=$ $4.32 \times 10^{-13} \mathrm{erg} \mathrm{s}^{-1} \mathrm{~cm}^{-2}$. The coronal abundance ratio of iron to oxygen is 0.67 during the flare (see Table 2), and from CHIANTI we calculate the ratio of the line emissivities of Fe xIII to the $\mathrm{O}$ vII triplet to be of the order of 0.25 in the temperature range $\log T=6.2-6.3$. From this, we derive an order-of-magnitude estimate of $F_{\mathrm{Fe} \mathrm{XIII}} \approx 10^{-15} \mathrm{erg} \mathrm{s}^{-1} \mathrm{~cm}^{-2}$, which is a little bit lower but given the uncertainties compatible with the flux computed from the optical spectra in Table 5.

The observation of the Fe xIII line during the larger flare in the third night seems to be counter-intuitive at first glance, since a flare should heat the corona and Fe XIII has its peak ionisation equilibrium at $T=1.6 \mathrm{MK}$. However, during the flare the emission measure for the low temperature component at $1.6 \mathrm{MK}$ is slightly higher than for quiecent state (or, given the errors, comparable to the emission measure during quiescence) and the iron abundance is similary higher, which is consistent with the forbidden Fe XIII line being seen during the flare. Moreover, for another M5.5 star, LHS 2076, the line was also observed only during a flare (Fuhrmeister et al. 2004). 


\section{Chromospheric and transition region properties of Proxima Centauri}

\subsection{Identification of chromospheric emission lines}

For the flare spectrum No. 9 in the blue arm and No. 76 in the red arm, respectively, we produced an emission line list containing 474 chromospheric emission lines, out of which 21 are from the red arm. In the blue arm, we analysed the flare spectrum directly, while in the red arm we subtracted the quiescent spectrum No. 74. The main part of spectrum No. 9 and spectrum No. 7 can be found in Fig. 13.

To access the line parameters, we fitted the background, central wavelength, half width $\sigma$, and line flux as free parameters using a Gaussian profile for every manually indicated emission line in a certain wavelength range. We typically chose $10 \AA$ as the wavelength range size for the fitting process, since the background variations for such a short wavelength interval are negligible. The line fit parameters including a (possible) identification can be found in Table 6 . We only show a few rows of the Table 6 in this paper as an example, while the whole table is provided only in electronic form.

The flux measurements given in the line catalogue may be affected by rather large errors mainly for the following reasons. First, the description with a Gaussian may give a poor fit quality if the lines have broad wings (and should be fitted with a Lorentzian/Voigt). Second, the background/quasicontinuum may be ill-defined. This is true for emission lines in a broader emission line wing, but also for emission cores of absorption lines and a variable background. Moreover, nine Balmer/Paschen and other lines could not be fitted at all because they are too broad or have an ill-defined background level (but the lines are clearly there). The absolute flux level is also estimated to have rather large errors as discussed in Sect. 2.1.

For the line identification, we generally used the catalogue of (Moore 1972). A few lines were identified using the NIST database $^{3}$. For the identifications from the Moore catalogue, the multiplet is also given in Table 6 . The spectra were first shifted to the rest wavelength for the identification process which should enable us to identify systematic line shifts caused by the flare.

Three lines could not be identified with any known line. Another 98 lines have only possible identifications for the following reasons: (1) the line was not found in the Moore catalogue, (2) the wavelength shift to the possible laboratory wavelength is large, (3) the line is blended severely with other lines, and (4) (most often) the line is the only one out of the multiplet. Since we excluded doubtful features from our line list, the list cannot be claimed to be complete, especially for weak lines. The identified lines belong in total to 32 different ions. The number of lines found for the individual ions is shown in Table 7. Statistically, all lines are blue-shifted by $0.01 \AA$.

We found Balmer lines up to number 19 during as well as outside the flare. The lines are only stronger during the flare. This indicates that the densities during the flare are not high enough to cause any Stark broadening of the lines, which would lead to the merging of higher order Balmer lines (Š́vestka 1972). This agrees well with the electron densities measured using the X-ray data (see Sect. 4.1.3), which have at most doubled during the flare compared to quiescence.

We compare the line list to that of the large flare on CN Leo (Fuhrmeister et al. 2008), which consists of 1143 chromospheric emission lines. The two catalogues overlap in the wavelength

\footnotetext{
3 Available online under

http://physics.nist.gov/PhysRefData/ASD/index.html
}

region from 3280 to $3850 \AA$. In this range, we compared the proportion of lines found in the $\mathrm{CN}$ Leo flare to those found in this event. While for Fe I, Ti I, Mn I, Ni I, and Cr I, the proportion of lines found in the $\mathrm{CN}$ Leo flare compared to the lines found in the Proxima Centauri flare is (partially much) larger than 2, for the single ionised species of the same elements the proportion is $1-1.5$. Moreover, nineteen lines in our present catalogue are not found in the CN Leo flare. These are $4 \mathrm{He}$ I lines, $2 \mathrm{Fe}$, 6 Fe II, 2 Zr II lines, 2 Co I, 1 V II, 1 Ti I, and $1 \mathrm{HI}$. This seems to indicate that the flare energy is deposited very effectively in the higher chromosphere/lower transition region, where helium and Balmer lines are formed, and also the lines of singly ionised metals should be formed.

\subsection{Line asymmetries}

During the flare, line asymmetries are found in both the blue and the red spectra. The Balmer lines, He I, and $\mathrm{Ca}$ II $\mathrm{K}$ show additional flux in the red wing. In the blue arm, we investigated the Balmer lines $\mathrm{H} \gamma, \mathrm{H} \delta$, and the Balmer lines at $3889 \AA$ and $3835 \AA$. We refrained from fitting $\mathrm{H} \epsilon$ because of the heavy blending with the $\mathrm{Ca}$ II $\mathrm{H}$ line. We also failed to fit the higher Balmer lines because of the extreme width of these lines during the flare, the blending with multiple metal lines, and the low line amplitudes of the very highest Balmer lines. Unfortunately, all He I lines in the blue arm are weak or blended and decay very fast, so that in the spectrum No. 10 after the flare peak spectrum No. 9, the helium lines have almost vanished. The Ca II $\mathrm{K}$ line being an unblended strong metal line shows a clear red wing asymmetry. No other metal lines have clear asymmetries. In the red arm, we find an asymmetry for the He I line at $6678 \AA$ in the flare peak spectrum No. 76 , while in spectrum No. 77 , the line has already faded too much to display any asymmetry. The other three helium lines in the red arm spectrum are either too weak or too complicated in their structure (blend with another helium line) to show unambiguous asymmetries. Hence, the only line for which we could study the asymmetries with higher time resolution of the red arm spectra is $\mathrm{H} \alpha$. This line shows indeed that the asymmetries have a strong time dependence that cannot be studied with the time resolution of the blue arm spectra. We fitted the line asymmetries with two Gaussians: one for the main (narrow) line component and one for the (broad) wing component. For all fits, we subtracted the quiescent spectrum directly before the flare. The shifts between the broad and the narrow Gaussian are listed in Table 8 for various lines and for the $\mathrm{H} \alpha$ line with higher time resolution in Table 9. Although the formal errors found in the fitting process for the line shifts are small, we assume a ten percent error for all the line shifts.

The high time resolution of $\mathrm{H} \alpha$ clearly shows that the asymmetries are a dynamical phenomenon as can be seen in Fig. 14. The asymmetries for the first secondary flare can be identified in the time map itself (elapsed time is about $1.3 \mathrm{~h}$ ). In addition, it can be seen in the top panel of Fig. 14, where the yellow shaded spectrum shows the most prominent asymmetry in the wing. The third peak in this light curve also shows broad wings in the $\mathrm{H} \alpha$ spectra, which are, however, weaker, thus could not be fitted. Starting with spectrum No. 149 (08:52:10 UT) and No. 150 with a blue asymmetry, the asymmetry turns to the red side in spectrum No. 152 and 153 (09:00:48 UT). These asymmetries can also be noted in the time map (elapsed time about $3.2 \mathrm{~h}$ in Fig. 14). While in the blue spectra there are only red asymmetries, in $\mathrm{H} \alpha$ there are also blue ones. Indeed for the entire integration time of the blue spectrum No. 9, there are mostly 


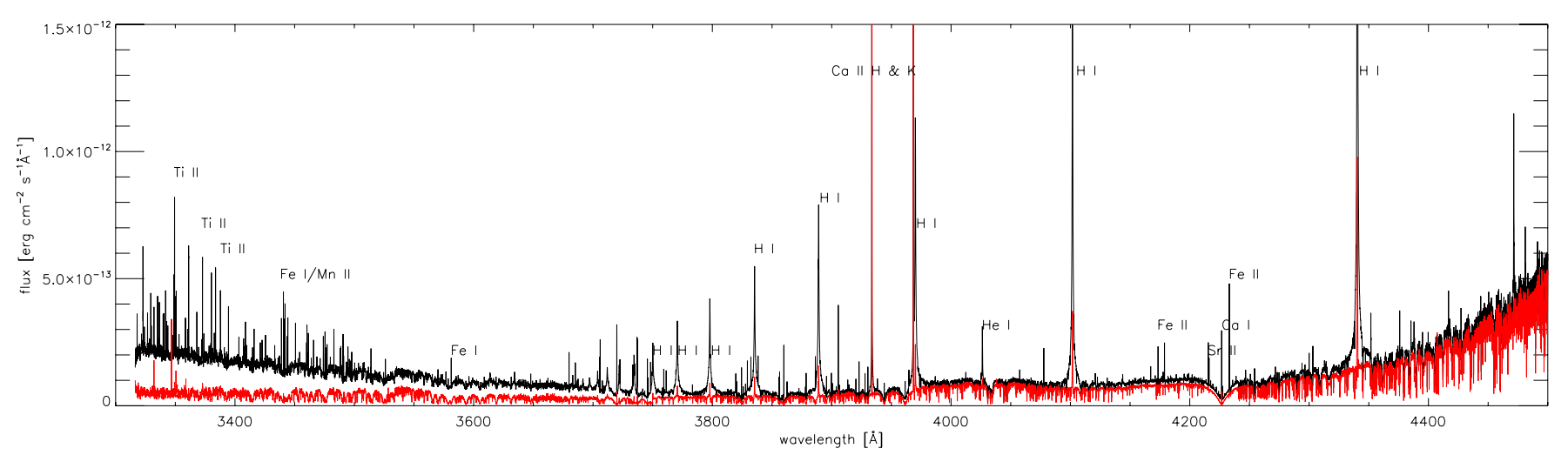

Fig. 13. Main part of the UVES blue flare (black) and quiescent (red/grey) spectrum. Several major chromospheric emission lines are identified in the plot.

Table 6. Ten rows of the line catalogue.

\begin{tabular}{|c|c|c|c|c|c|c|c|}
\hline $\begin{array}{l}\text { Central wavelength } \\
{[\AA]}\end{array}$ & $\begin{array}{c}\text { Half width } \\
{[\AA]}\end{array}$ & $\begin{array}{c}\text { Flux }^{a} \\
{\left[\mathrm{erg} \mathrm{s}^{-1} \mathrm{~cm}^{-2}\right]}\end{array}$ & $\begin{array}{c}\text { Catalogued wavelength } \\
{[\AA]} \\
\end{array}$ & Ion & Multiplet & Id flag & Comment \\
\hline 3495.35 & 0.06 & $8.70 \times 10^{-15}$ & 3495.285 & $\mathrm{Fe}_{\mathrm{I}}$ & 238 & 2 & blend 3495.370 CrII 2 \\
\hline 3495.82 & 0.04 & $1.25 \times 10^{-14}$ & 3495.831 & Mn II & 3 & 0 & \\
\hline 3496.80 & 0.03 & $1.01 \times 10^{-14}$ & 3496.814 & Mn II & 3 & 0 & \\
\hline 3497.09 & 0.04 & $3.93 \times 10^{-15}$ & 3497.115 & $\mathrm{Fe}_{\mathrm{I}}$ & 78 & 2 & em \\
\hline 3497.51 & 0.03 & $7.56 \times 10^{-15}$ & 3497.536 & Mn II & 3 & 0 & em \\
\hline 3497.83 & 0.03 & $1.21 \times 10^{-14}$ & 3497.843 & $\mathrm{Fe}_{\mathrm{I}}$ & 6 & 0 & em \\
\hline 3500.33 & 0.04 & $8.34 \times 10^{-15}$ & 3500.340 & Ti II & 6 & 0 & \\
\hline 3500.87 & & & 3500.852 & $\mathrm{Ni}$ I & 6 & 0 & em, line not fitable \\
\hline 3503.46 & 0.05 & $7.53 \times 10^{-15}$ & 3503.474 & $\mathrm{Fe}_{\text {II }}$ & 4 & 0 & \\
\hline 3504.89 & 0.02 & $1.23 \times 10^{-15}$ & 3504.890 & Ti II & 88 & 0 & \\
\hline
\end{tabular}

Notes. The whole table is accessible electronically at the CDS. ${ }^{(a)}$ As discussed in Sect. 2.1, the absolute errors for the given fluxes are about a factor of two.

Table 7. Chromospheric emission line identifications.

\begin{tabular}{ll|ll}
\hline \hline Ion & No. lines $^{1}$ & Ion & No. lines $^{1}$ \\
\hline $\mathrm{H}_{\text {I }}$ & $19(19)$ & $\mathrm{Mg}_{\text {I }}$ & $10(6)$ \\
$\mathrm{He}_{\text {I }}$ & $14(6)$ & $\mathrm{K}_{\text {II }}$ & $1(0)$ \\
$\mathrm{Li}_{\text {II }}$ & $1(0)$ & $\mathrm{Ca}_{\text {I }}$ & $1(0)$ \\
$\mathrm{Sc}_{\text {II }}$ & $7(4)$ & $\mathrm{Ca}_{\text {II }}$ & $7(7)$ \\
$\mathrm{Ti}_{\text {I }}$ & $3(0)$ & $\mathrm{C}_{\text {I }}$ & $5(4)$ \\
$\mathrm{Ti}_{\text {II }}$ & $58(53)$ & $\mathrm{O}_{\text {I }}$ & $3(3)$ \\
$\mathrm{V}_{\text {II }}$ & $2(0)$ & $\mathrm{Ne}_{\text {I }}$ & $1(0)$ \\
$\mathrm{Cr}_{\text {I }}$ & $9(7)$ & $\mathrm{Ne}_{\text {II }}$ & $1(0)$ \\
$\mathrm{Cr}_{\text {II }}$ & $30(26)$ & $\mathrm{Al}_{\text {I }}$ & $2(2)$ \\
$\mathrm{Mn}_{\text {I }}$ & $4(3)$ & $\mathrm{Si}_{\text {I }}$ & $2(0)$ \\
$\mathrm{Mn}_{\text {II }}$ & $9(8)$ & $\mathrm{Si}_{\text {II }}$ & $4(3)$ \\
$\mathrm{Fe}_{\text {I }}$ & $182(148)$ & $\mathrm{Cl}_{\text {II }}$ & $1(0)$ \\
$\mathrm{Fe}_{\text {II }}$ & $45(35)$ & $\mathrm{Ce}_{\text {II }}$ & $1(0)$ \\
$\mathrm{Co}_{\text {I }}$ & $5(2)$ & $\mathrm{Sr}_{\text {II }}$ & $2(2)$ \\
$\mathrm{Ni}_{\text {I }}$ & $31(28)$ & $\mathrm{Zr}_{\text {II }}$ & $3(2)$ \\
$\mathrm{Ni}_{\text {II }}$ & $5(5)$ & $\mathrm{Y}_{\text {II }}$ & $2(0)$ \\
\hline
\end{tabular}

Notes. ${ }^{(1)}$ In brackets we indicate the number of secure identifications.

blue-shifts for $\mathrm{H} \alpha$; only for red spectrum No. 76 corresponding to the first few minutes exposure time of spectrum No. 9 there is a red shift (the spectrum No. 9 corresponds to elapsed time from 0.4 to $0.7 \mathrm{~h}$ in Fig. 14). Since the amplitude decays exponentially, the conditions at the beginning of the spectrum should dominate the spectrum. Therefore, blue spectrum No. 9 and spectrum No. 76 yield similar red shifts. After red spectrum
Table 8. Line shifts of the broad line component for diverse lines from the flare-only spectra.

\begin{tabular}{|c|c|c|c|}
\hline$\overline{\text { Line }}$ & $\begin{array}{c}\text { Velocity } \\
{\left[\mathrm{km} \mathrm{s}^{-1}\right]} \\
\text { spec. No. } 8(75) \\
05: 47: 14\end{array}$ & $\begin{array}{c}\text { Velocity } \\
{\left[\mathrm{km} \mathrm{s}^{-1}\right]} \\
\text { spec. No. } 9(76) \\
06: 14: 39\end{array}$ & $\begin{array}{c}\text { Velocity } \\
{\left[\mathrm{km} \mathrm{s}^{-1}\right]} \\
\text { spec. No. 10(77) } \\
\text { 06:45:12 }\end{array}$ \\
\hline $\mathrm{H} \gamma(4340 \AA)$ & - & 4.8 & 12.4 \\
\hline $\mathrm{H} \delta(4101 \AA)$ & 3.9 & 4.9 & 10.2 \\
\hline Н г $3889 \AA$ & - & fit ambiguous & 13.1 \\
\hline H I $3835 \AA$ & 2.1 & fit ambiguous & 9.4 \\
\hline Ca II K & - & 5.1 & 21.4 \\
\hline He I $6678 \AA$ & - & 6.0 & - \\
\hline
\end{tabular}

No. 80 (corresponding to an elapsed time of about $0.6 \mathrm{~h}$ ), the asymmetries and large wings disappear - but re-appear in red spectrum No. 97-102 (corresponding to an elapsed time of about 1.1 to $1.3 \mathrm{~h}$ in Fig. 14). This agrees with the red wing asymmetries in blue spectrum No. 10, although the asymmetry in the red arm is invisible for the whole integration time of this spectrum. That the time scales on which the asymmetries evolve can be very short can be seen in red spectrum No. 75 and blue spectrum No. 8. While the red spectrum shows a clear blue-shift, in the blue spectrum the lines either cannot be fitted or show no real line shifts, but do display broad wings (see Tables 8, 9).

We interpret all these line shifts as manifestations of mass motions in the chromosphere as e.g. Fuhrmeister et al. (2008). The high velocity blue-shift at the flare onset corresponds to 


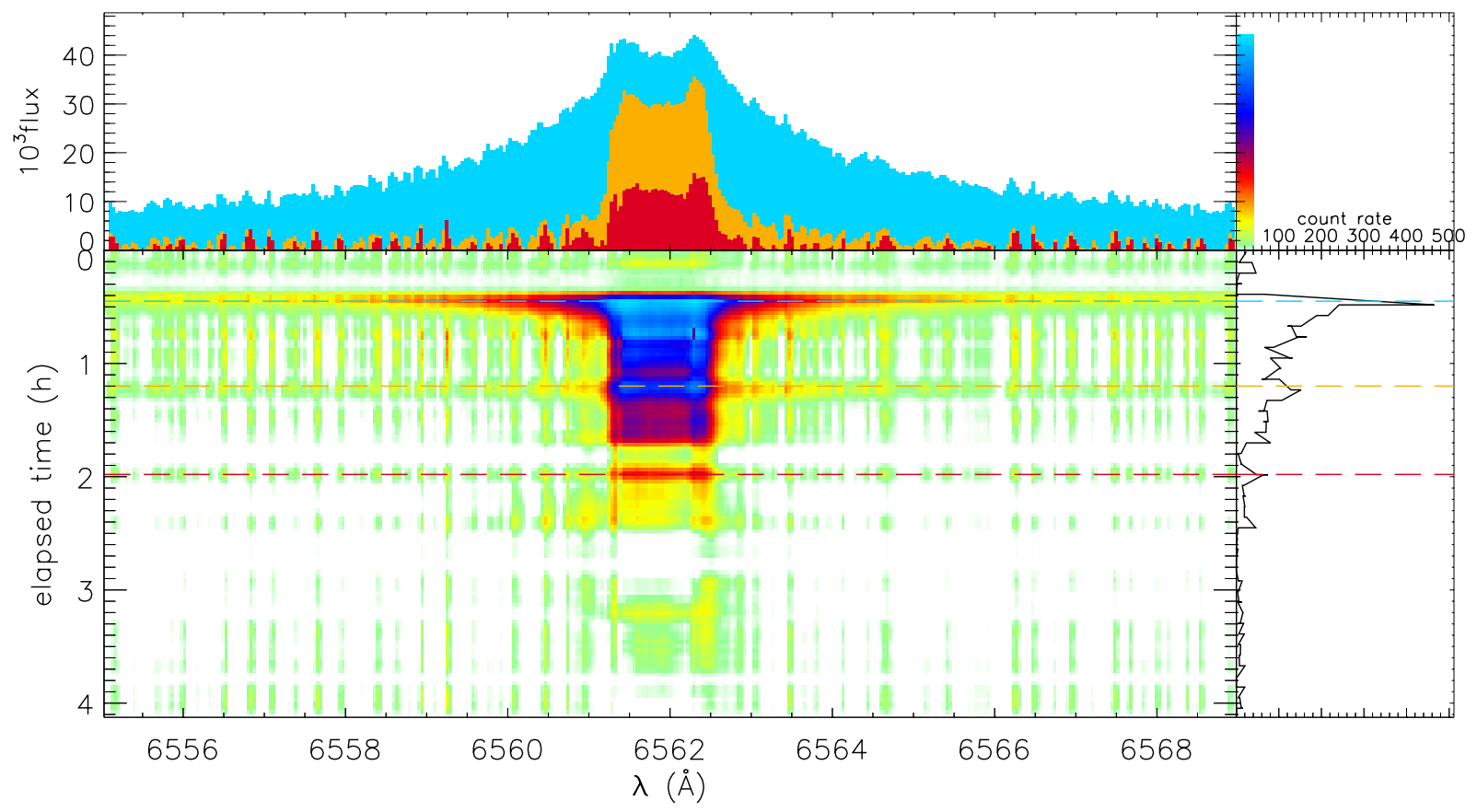

Fig. 14. The $\mathrm{H} \alpha$ line variations in time with the quiescence spectrum No. 74 subtracted. The top panel shows three individual spectra as marked by the horizontal dashed lines in the time map below. On the right hand panel, we show the light curve of the integrated flux of the quiescence subtracted spectrum for the shown wavelength region. The time is measured in elapsed time since 5:50 UT.

Table 9. Line shifts of the broad line component for the $\mathrm{H} \alpha$ line from the flare-only spectra.

\begin{tabular}{lccccc}
\hline \hline $\begin{array}{l}\text { Spectrum } \\
\text { No. }\end{array}$ & $\begin{array}{c}\text { Start time } \\
(\mathrm{UT})\end{array}$ & $\begin{array}{c}\text { Velocity } \\
{\left[\mathrm{km} \mathrm{s}^{-1}\right]}\end{array}$ & $\begin{array}{c}\text { Spectrum } \\
\text { No. }\end{array}$ & $\begin{array}{c}\text { Start time } \\
(\mathrm{UT})\end{array}$ & $\begin{array}{c}\text { Velocity } \\
{\left[\mathrm{km} \mathrm{s}^{-1}\right]}\end{array}$ \\
\hline 75 & $06: 12: 16$ & -44.3 & 97 & $06: 59: 50$ & 8.7 \\
76 & $06: 14: 29$ & 5.3 & 98 & $07: 02: 00$ & 13.7 \\
77 & $06: 16: 37$ & -10.5 & 99 & $07: 04: 09$ & 13.7 \\
78 & $06: 18: 48$ & -9.6 & 100 & $07: 06: 19$ & 33.7 \\
79 & $06: 20: 57$ & -5.9 & 101 & $07: 08: 28$ & 32.9 \\
80 & $06: 23: 06$ & 1.4 & 102 & $07: 10: 37$ & 3.7 \\
\hline
\end{tabular}

evaporation of chromospheric material, while during the decay phase raining down as well as rising material can be found.

\subsection{Theoretical modelling of the flare with PHOENIX}

We modelled the flare spectra with theoretical PHOENIX chromospheric flare spectra. These spectra were originally computed to fit the mega-flare on CN Leo (Fuhrmeister et al. 2010). The stars CN Leo and Proxima Centauri have similar photospheric properties. While for Proxima Centauri, $T_{\text {eff }}$ is about $3100 \mathrm{~K}$, for CN Leo a $T_{\text {eff }}$ of $2900 \mathrm{~K}$ was used for the underlying photosphere in the model calculation. Short \& Doyle (1998) studied the influence of the underlying photosphere on a quiescent chromospheric model and found that a difference of $200 \mathrm{~K}$ in $T_{\text {eff }}$ leads to uncertainties in the parameters of the column mass at the onset of the transition region as well as the column mass of the temperature minimum of about 0.3 dex. The finest step in our flaring model grid is 0.2 dex. Since we use a photosphere with a lower $T_{\text {eff }}$ than is realistic, we should infer a too high column mass for the onset of the transition region and the temperature minimum. Details about the model construction can be found in Fuhrmeister et al. (2010). In this paper, we constructed the flaring model as a linear combination of a quiescent spectrum observed directly before the flare and a flaring model spectrum.
Table 10. Mean best-fit flare model parameters in time.

\begin{tabular}{lccccc}
\hline \hline $\begin{array}{l}\text { Spec. } \\
\text { no. }\end{array}$ & $\begin{array}{c}T_{\text {chrom }} \\
{[\mathrm{K}]}\end{array}$ & $\begin{array}{c}\log \\
\text { cmas } s_{\text {Tchrom }}\end{array}$ & $\begin{array}{c}\log \\
\text { cmass }_{\text {Tmin }}\end{array}$ & grad TR & $\begin{array}{c}\text { Filling } \\
\text { factor }\end{array}$ \\
\hline 76 & $8400 \pm 300$ & $-2.5 \pm 0.2$ & $-0.3 \pm 0.2$ & $11.0 \pm 0.1$ & $2.0 \pm 0.5$ \\
77 & $8400 \pm 200$ & $-2.5 \pm 0.1$ & $-0.3 \pm 0.2$ & $11.1 \pm 0.3$ & $2.0 \pm 0.1$ \\
78 & $8300 \pm 200$ & $-2.7 \pm 0.3$ & $-0.4 \pm 0.0$ & $11.1 \pm 0.3$ & $2.0 \pm 0.1$ \\
80 & $8200 \pm 300$ & $-2.7 \pm 0.3$ & $-0.4 \pm 0.2$ & $11.1 \pm 0.3$ & $2.0 \pm 0.1$ \\
82 & $8300 \pm 300$ & $-2.9 \pm 0.2$ & $-0.9 \pm 0.3$ & $11.0 \pm 0.4$ & $4.0 \pm 0.1$ \\
\hline 95 & $8000 \pm 100$ & $-3.0 \pm 0.1$ & $-1.1 \pm 0.3$ & $11.0 \pm 0.4$ & $6.0 \pm 1.0$ \\
96 & $8400 \pm 300$ & $-2.6 \pm 0.3$ & $-0.2 \pm 0.2$ & $11.0 \pm 0.1$ & $2.0 \pm 0.8$ \\
97 & $8400 \pm 300$ & $-2.5 \pm 0.2$ & $-0.3 \pm 0.2$ & $11.0 \pm 0.1$ & $1.5 \pm 0.4$ \\
98 & $8400 \pm 300$ & $-2.7 \pm 0.2$ & $-0.3 \pm 0.2$ & $11.1 \pm 0.3$ & $1.5 \pm 0.4$ \\
99 & $8400 \pm 300$ & $-2.7 \pm 0.2$ & $-0.3 \pm 0.2$ & $11.1 \pm 0.3$ & $1.5 \pm 0.4$ \\
101 & $8200 \pm 300$ & $-3.0 \pm 0.1$ & $-1.1 \pm 0.5$ & $10.9 \pm 0.3$ & $4.0 \pm 0.1$ \\
\hline 9 & $8500 \pm 100$ & $-2.5 \pm 0.2$ & $-0.3 \pm 0.2$ & $11.0 \pm 0.4$ & $0.8 \pm 0.1$ \\
10 & $8400 \pm 300$ & $-2.9 \pm 0.2$ & $-0.8 \pm 0.3$ & $11.0 \pm 0.1$ & $0.9 \pm 0.1$ \\
\hline
\end{tabular}

For the red and the blue arm respectively, we then used a global normalisation of the spectra and a grid of different filling factors of the flare model spectra to the quiescent observed spectra to help us find the best fit to the observed flare spectra. The filling factors used for the quiescent spectra are $0.85,0.90$, $0.93,0.95,0.96,0.97,0.98,0.99,0.995$, and 0.999 . The best fit was found using a $\chi^{2}$ analysis again following Fuhrmeister et al. (2010). The mean best-fit parameters for the five best-fit flare models can be found in Table 10 with the standard deviation of the mean. Examples of the temperature structure of the best-fit models for a selection of different spectra can be found in Fig. 15. For a small wavelength range in the blue, a comparison between the observed spectrum and best-fit model spectrum can be found in Fig. 16.

The model parameters of the fitting process from the blue and the red arm agree well with each other with both exhibiting a shift towards flare models further out in the atmosphere later in the decay phase. For red models later than No. 82, no meaningful fit could be found with many models exhibiting nearly the same $\chi^{2}$. Even later during the secondary flare phase, a fitting of the flaring models also turned out to be possible again, starting at red 


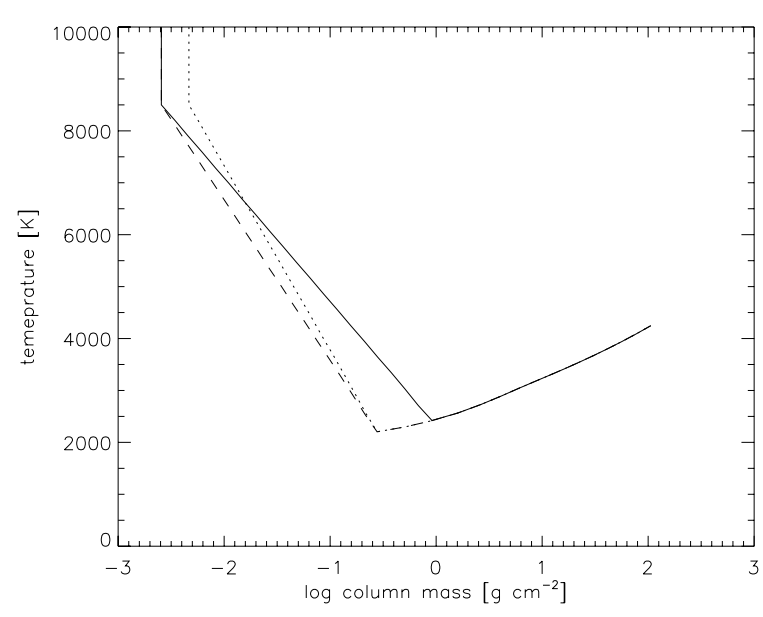

Fig. 15. Example of the temperature structure of the best-fit flare models for different spectra: black line: red spectrum No. 76 (flare onset); dotted line: red spectrum No. 97 (secondary flare); dashed line: blue spectrum No. 9.

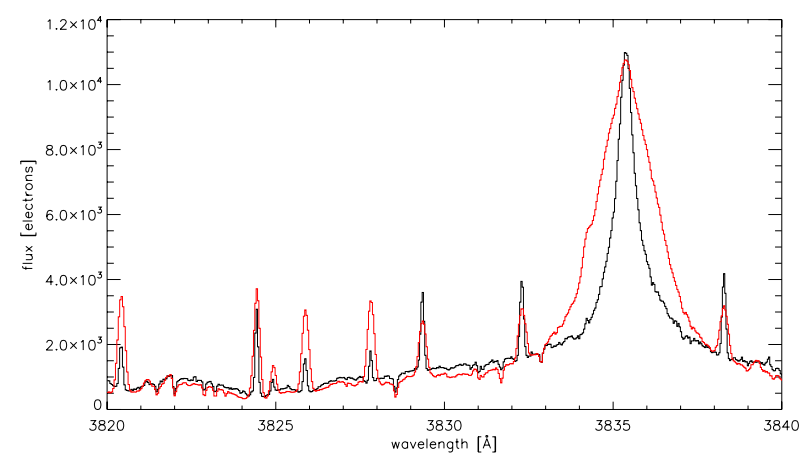

Fig. 16. Exemplary comparison between observed spectrum (black) and our best-fit model spectrum (red) for blue spectrum No. 9.

spectrum No. 95. The secondary flare can be seen in the models as a re-shift to higher-pressure chromospheric flare models. For the higher-pressure models, a higher temperature for the onset of the transition region is also found.

The only disagreement between model parameters can be found for the filling factor. Comparing red spectrum No. 76 to blue spectrum No. 9 shows that the blue spectrum has a lower filling factor. This may indicate that there is an error in the normalisation, which was done for the red arm where the influence of the flare on the continuum should be small and then applied to the blue arm. Another possibility of the different filling factors is a height dependence of the filling factor. The blue arm with its wealth of metal lines should trace the mid-chromosphere, while the red arm with the He I, Balmer, and Paschen lines should trace the upper chromosphere. The filling factor found for the red spectra models of about two percent agrees well with that found by the EM-T diagnostics from the X-ray data, which is three percent (see Sect. 4.2).

\section{Discussion}

The multi-wavelength character of the observations allows us to characterise general behaviour and energetics of the flare for various wavelength bands, hence different temperature regimes and also cross-check these using different instruments.
Table 11. Various fluxes and flare energetics for different wavelength regimes.

\begin{tabular}{lccc}
\hline \hline Wavelength band & Instrument & $\begin{array}{c}\text { Flux }^{a} \\
{\left[\mathrm{erg} \mathrm{s}^{-1} \mathrm{~cm}^{-2}\right]}\end{array}$ & $\begin{array}{c}\text { Energy } \\
{[\mathrm{erg}]}\end{array}$ \\
\hline chrom. em. lines & UVES blue arm & $1.2 \times 10^{-11}$ & $8.5 \times 10^{28}$ \\
$\mathrm{H} \alpha$ emission & UVES red arm & $2.0 \times 10^{-12}$ & $1.6 \times 10^{28}$ \\
blue excess em. & UVES blue arm & $5.3 \times 10^{-10}$ & $3.8 \times 10^{30}$ \\
$(3300-3600 \AA)$ & & $2.2 \times 10^{-11}$ & \\
X-rays & PN+RGS XMM & $\pm 0.2 \times 10^{-11}$ & $1.9 \times 10^{29}$ \\
$(0.2-10 \mathrm{keV})$ & & \\
\hline
\end{tabular}

Notes. ${ }^{(a)}$ The errors in the UVES fluxes are about a factor of two (see Sect. 2.1).

\subsection{Flare energetics and excess emission}

The flare exhibited a broadband excess emission in the far blue of the UVES spectral arm, which can be easily identified in Fig. 13. A similar excess was found for larger flares and could in these cases be ascribed to blackbody emission (Fuhrmeister et al. 2008; Hawley \& Fisher 1992). In the flare observed here, it was impossible to fit the excess emission with a blackbody model. We nevertheless calculated the excess flux, noting that the shape of the spectrum suggests that the bulk of the broadband emission is not covered by the UVES spectrum. We integrated the flare spectrum up to $3600 \AA$ and subtracted (1) the quiescent flux, (2) the general flux increase seen in the blue arm during the flare, and (3) the chromospheric line flux in this wavelength interval for the flux excess calculation. We also calculated the total of the chromospheric line fluxes in the blue arm and in $\mathrm{H} \alpha$. We compared the $\mathrm{H} \alpha$ line flux to the value one obtains using the $\chi$ method introduced by West \& Hawley (2008), which is based only on the equivalent width of the line, the bolometric luminosity, and the distance of the star (so one needs no absolute flux calibration). Using the $\chi$ value of $0.176 \times 10^{-4}$ for an M 6 dwarf, $L_{\text {bol }}$ of $6.7 \times 10^{30} \mathrm{erg} \mathrm{s}^{-1}$, and a measured peak EW of 3.4, we evaluated the same flux of $2.0 \times 10^{-12} \mathrm{erg} \mathrm{s}^{-1} \mathrm{~cm}^{-2}$ as in our flux calibration (or $1.8 \times 10^{-12} \mathrm{erg} \mathrm{s}^{-1} \mathrm{~cm}^{-2}$ using $L_{\mathrm{bol}}$ of $6.0 \times 10^{30} \mathrm{erg} \mathrm{s}^{-1}$ ). The various fluxes can be found in Table 11 together with the resulting energies assuming a filling factor for the flare of two percent for the time range of the blue UVES spectrum No. 9 from 6:14 to 6:45 UT.

\subsection{Ho self-absorption}

A comparison to the flux-calibrated spectrum of Proxima Centauri presented by Cincunegui et al. (2007) shows that the $\mathrm{H} \alpha$ line amplitude in our spectra is extremely small during the whole three days of our observations. The line amplitude during the flare is comparable to the line amplitude of the spectrum from Cincunegui et al. (2007), which was taken during a quiescent state. This indicates that Proxima Centauri's chromosphere was generally in a low activity state during the observations. The occurring flare enhanced the $\mathrm{H} \alpha$ line flux only to a level that can also be found during a quiescent state (at other times). This fits well with the relatively low X-ray flux found in our observations (see Sect. 3.1).

The $\mathrm{H} \alpha$ line is heavily self-absorbed during our observations. This self-absorption is in most cases asymmetric. The line peak at the red side is higher than at the blue; this peak asymmetry should not be confused with the wing asymmetry described in Sect. 5.2 of this paper. Two examples can easily be noted in 
the "flare only" spectra shown in the top panel of Fig. 14: while for the flare spectrum the two peaks are about of the same height and the line centre therefore symmetric, for the two spectra from the decay phase the red peak is higher than the blue peak. We note that the spectra of Cincunegui et al. (2007) have lower spectral resolution hiding a possible self-reversal. We therefore compared our data to processed HARPS data from the ESO archive facility ${ }^{4}$, which cover the spectrum of Proxima Centauri from 3782 to $6912 \AA$, in the time from 2004 to 2007 . These spectra have not yet been published and show a wide variation in the $\mathrm{H} \alpha$ line amplitude, with the spectra from Cincunegui et al. (2007) at the high end of measured $\mathrm{H} \alpha$ line amplitudes and our observations at the low end. Like our spectra the HARPS spectra exhibit in most cases a self-reversal with more flux in the red side peak.

We checked the red peak asymmetries using our models, which also display $\mathrm{H} \alpha$ self-absorption at the line centre that is in all cases symmetric. This would be expected for onedimensional (1D) chromospheric models without mass motion, where the self-reversal is a pure non-local thermal equilibrium (NLTE) effect. Allred et al. (2006) computed 1D hydrodynamical models of $\mathrm{M}$ dwarf flares. For various evolutionary stages of the flare in their simulations, the $\mathrm{H} \alpha$ line profiles show the red peak asymmetries also observed in our and the HARPS spectra. In the hydro-dynamical models, the peak asymmetry is caused by mass motions during the simulated flare. It appears reasonable to assume that similar mass motions are also common in the quiescent state to explain the observed peak asymmetries.

\subsection{Magnetic field}

The dip in the magnetic field coincides in time with the flare in the third night. We estimated the energetics as a consistency check, in case a physical connection were possible. The level of the magnetic field changes from $\approx 300 \pm 100 \mathrm{G}$ just before the flare to $\approx 100 \pm 100 \mathrm{G}$ at the time the flare starts in the optical. So, the nominal change in mean magnetic field is $200 \mathrm{G}$, although the errors are very large. As a consistency check however, we can calculate that this corresponds to a released magnetic energy density of

$\eta=\frac{B^{2}}{8 \pi}=1.6 \times 10^{3} \mathrm{erg} \mathrm{cm}^{-3}$.

In Sect. 4.2, we calculated the flaring volume to be $V_{\text {flare }} \approx$ $6.25 \times 10^{29} \mathrm{~cm}^{3}$, yielding a total released magnetic energy of $1.0 \times 10^{33} \mathrm{erg}$. We computed the total X-ray energy released during the first $6.5 \mathrm{ks}$ of the flare to be $1.5 \times 10^{31} \mathrm{erg}$ by integrating the flare and quiescent spectral models from Table 2 over the energy range $0.2-10 \mathrm{keV}$ and calculating the difference between the two. This is two orders of magnitude less than our estimate for the total released magnetic energy. However, since the detailed loop configuration is not known and we simply estimated the flaring volume as $V=L^{3}$ with $L$ being the loop half length, the volume itself can only be an order-of-magnitude estimate. Therefore, we interpret the derived numbers as a general confirmation that the emitted X-ray energy during the flare is within the energy budget of the theoretically released magnetic energy. The question, of whether the dip in magnetic field is really physically connected to the flare must unfortunately remain unsolved.

Other flare stars of similar spectral type do show higher quiescent activity levels. Although magnetic field measurements of

\footnotetext{
${ }^{4}$ Based on data obtained from the ESO Science Archive Facility from program 072.C-0488(E).
}

mid and late-type $\mathrm{M}$ dwarfs are still quite rare, there is a time series measurement for the M5.5 star CN Leo (Reiners et al. 2007) with mean magnetic field of $2.2 \mathrm{kG}$ covering a large flare. A dip in $B f$ at the time of the flare is also seen in these data, although there are other field variations of similar amplitude that are not associated with flares. Thus, it remains unclear whether the change in magnetic field is associated with the flare.

Reiners et al. (2007) found a correlated change in the distributions of magnetic field $B f$ and $\mathrm{H} \alpha$ emission in their data of CN Leo. We searched for a similar correlation in our data, but found no one, which is unsurprising as the magnetic field changes are only small. We note that the $\mathrm{H} \alpha$ emission during the course of these observations are quite low (see Sect. 6.2) corresponding to a weak magnetic field. Unfortunately, the observation of Reiners \& Basri (2008), which found a stronger magnetic field for Proxima Centauri (as described in Sect. 3.3) does not include the $\mathrm{H} \alpha$ line.

\subsection{Line asymmetries}

Line asymmetries during flares have often been found in high resolution spectra mostly for $\mathrm{H}_{\mathrm{I}}$ and $\mathrm{He}_{\mathrm{I}}$ lines. Examples are red line asymmetries for a large flare on LHS 2034 (M6.5) (Fuhrmeister et al. 2005), blue line asymmetries for a flare on AT Mic (M4) (Gunn et al. 1994), and red line asymmetries for flares on AD Leo (M3.5Ve) (Crespo-Chacón et al. 2006, for further examples see also references therein). For the CN Leo mega-flare described by Fuhrmeister et al. (2008), bluewing as well as red-wing asymmetries have also been found. Asymmetries were normally ascribed by these authors to mass motions, which were partially modelled with multiple kernels and compared to the chromospheric downward condensations (CDCs) observed in the Sun in the case of the red asymmetries. During the CN Leo flare, asymmetries in the wings of Ca II lines were also found for the first time. Surprisingly, we found asymmetries in the $\mathrm{Ca}$ II $\mathrm{H}$ and $\mathrm{K}$ line for this much smaller flare on Proxima Centauri, though Crespo-Chacón et al. (2006) found that larger flares have stronger asymmetries and found no asymmetry in the $\mathrm{Ca}$ II $\mathrm{H}$ and $\mathrm{K}$ line in their flare data (their longest flares lasted about $30 \mathrm{~min}$ ).

In contrast to the $\mathrm{CN}$ Leo mega-flare we also found blue wing asymmetries during flare decay and for the first time a reappearance of the asymmetries during the secondary flare phase. The vanishing and re-appearance of the broad wings combined with the asymmetries of the $\mathrm{H} \alpha$ line coincides with the secondary flare process seen in the X-ray light curve (see Fig. 3 and Table 9). When the secondary flare started, the broad component vanished, but reappeared some time later. The reappearance of the broad wings can also be identified in the $\mathrm{H} \alpha$ light curve (see Fig. 3) as the peak after the main flare peak. The data again illustrates the dynamic behaviour of the asymmetry phenomenon, which provides additional evidence of mass motions.

\subsection{Theoretical modelling}

The clear secondary flare found by the flare models for the chromosphere strengthens our confidence in this approach of mixing quiescent observations with flaring model spectra. However, the general caveats of the method presented by Fuhrmeister et al. (2010) still apply: the 1D models show (for simplicity reasons) a linear temperature rise, which can explain part of the difficulties in fitting large wavelength regions with the same model spectrum equally well. Moreover, the 1D models are a very 


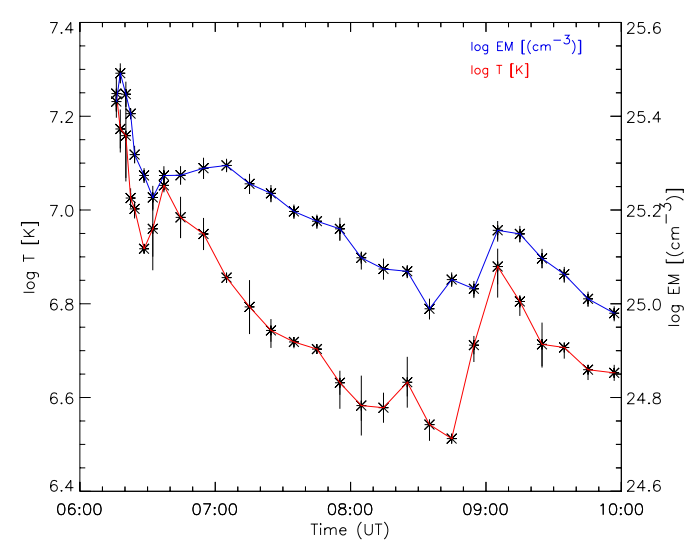

Fig. 17. Temporal evolution of flare temperature and emission measure.

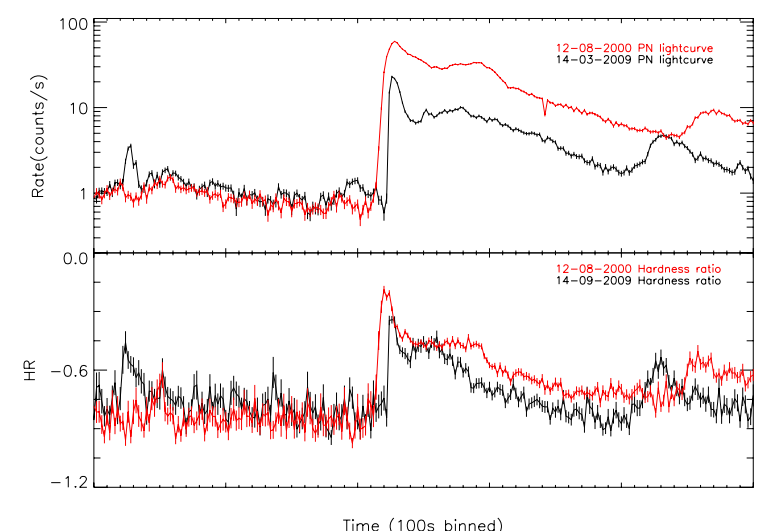

Fig. 18. Light curves with $100 \mathrm{~s}$ binning and hardness ratio of flares observed on Proxima Centauri by XMM-Newton on 12 August 2001 (red) and 14 March 2009 (black), respectively.

rough description of the horizontally as well as vertically highly complex temperature pattern (including shocks) of the (solar) chromosphere. Therefore, more physical chromospheric models should include hydrodynamic simulations and dynamic ionisation in either $1 \mathrm{D}$ or $3 \mathrm{D}$.

\subsection{Secondary flare events}

The nature of this flare with its two secondary events following the first peak in the light curve raises the question of what kind of magnetic structure we are dealing with. There are two possibilities: it could be a single loop that experiences two additional heat pulses after the initial flare, or an arcade structure where several loops light up in sequence. The heat pulse refers to the process where the electron beam is accelerated in the coronal part of the magnetic flare loop, then propagates along the magnetic field lines down to the chromosphere, where the electrons are fully thermalised. A heat pulse represents the heating of the atmosphere by the electron beam. Reale et al. (2004) interpreted an earlier long-duration flare light curve of Proxima Centauri as being produced by two loops flaring in sequence, since the coronal temperature during the second peak was flattopped and could not be adequately explained by a second heat pulse in the same loop. In our data however, the temperature and emission measure evolution follows the trend seen in the light curves closely as can be seen comparing Figs. 17 and 3.
From our analysis of the $E M-T$ diagram for this flare, we derive very similar $E M-T$ slopes for all three flare decays, each one indicating that a moderate amount of continuous reheating is present. The thus derived loop lengths are of the same order of magnitude. Even if the errors are rather large, there is a decreasing trend discernible within these three lengths; the lengths derived from the second and third peaks are comparable, while the length derived from the first peak seems to be larger by a factor of roughly two. This, and that the amount of reheating during the decays is very similar, suggests that we may see a series of similar loops in an arcade lighting up in sequence. However, owing to the large errors in the loop lengths, the possibility of a single loop with several heat pulses cannot be ruled out completely. A comparison of the X-ray and hardness ratio light curves for the mega-flare observed by Reale et al. (2004) and Güdel et al. (2004) and the flare presented here can be found in Fig. 18. The similarity of the light curves of the two events (despite the different count rates) is quite remarkable.

\section{Summary and conclusions}

We have presented multi-wavelength observations of the different activity stages of Proxima Centauri with particular emphasis on a long duration flare. We have tried to construct an overall picture of the atmosphere from the photosphere to the corona. We have determined a low average magnetic field, corresponding to low average X-ray and $\mathrm{H} \alpha$ emission, with the latter being heavily self-absorbed. For the flare, we have compiled a chromospheric emission line list and found asymmetries in the broad wings of Balmer, $\mathrm{He}$, and $\mathrm{Ca}$ II $\mathrm{H} \& \mathrm{~K}$ lines, which we ascribe to mass motions, which could explain coronal abundance changes. From the X-ray data, we have determined coronal densities, abundances, emission measures, and temperatures. The higher emission measure of the low temperature component and the higher Fe abundance during the flare fit well with the detection of a forbidden optical Fe XIII line during this time. The flare light curve is similar to the mega-flare for the same star described by Reale et al. (2004) and Güdel et al. (2004) exhibiting two bumps during the decay phase. The secondary flares are of a similar loop length as the main flare, show line asymmetries, and chromospheric flare modelling shows that the first one exhibits similar chromospheric parameters as the main one. All this indicates that the events are not independent of each other, but result from the same loop or at least an arcade with several arcade loops igniting consecutively. This seems to be a typical flare scheme for Proxima Centauri given, that it has been observed for the second time, and Reale et al. (2004) noted the similarity to solar flares. In contrast to Proxima Centauri, CN Leo, another well-observed M5.5 but more active dwarf never showed a comparable flare cascade during a long-duration flare. On the other hand, another highly active dM4.5e star, YZ CMi, also exhibited a series of secondary flares during a white light mega-flare as described by Kowalski et al. (2010b,a), who also speculated that the flare on YZ CMi originated in a complex arcade with a sequence of reconnecting loops. If these interpretations are correct, mid-type $\mathrm{M}$ dwarfs seems to have a flaring loop geometry that may be similar to the Sun, which will help to place constraints on turbulent dynamo theories.

Acknowledgements. S.L. acknowledges funding by the DFG in the framework of RTG 1351. K.P. acknowledges funding under project number DLR 50OR0703. N.R. acknowledges financial support by the DLR under project no. $50 \mathrm{OR} 1002$. 


\section{References}

Allred, J. C., Hawley, S. L., Abbett, W. P., \& Carlsson, M. 2006, ApJ, 644, 484 Arnaud, K. A. 1996, in Astronomical Data Analysis Software and Systems V, ed. G. H. Jacoby, \& J. Barnes, ASP Conf. Ser., 101, 17

Audard, M., Güdel, M., Sres, A., Raassen, A. J. J., \& Mewe, R. 2003, A\&A, 398, 1137

Ballester, P., Modigliani, A., Boitquin, O., et al. 2000, The Messenger, 101, 31

Berger, E., Gizis, J. E., Giampapa, M. S., et al. 2008, ApJ, 673, 1080

Brinkman, A. C., Behar, E., Güdel, M., et al. 2001, A\&A, 365, L324

Browning, M. K. 2008, ApJ, 676, 1262

Chabrier, G., \& Baraffe, I. 1997, A\&A, 327, 1039

Cincunegui, C., \& Mauas, P. J. D. 2004, Boletin de la Asociacion Argentina de Astronomia La Plata Argentina, 47, 114

Cincunegui, C., Díaz, R. F., \& Mauas, P. J. D. 2007, A\&A, 461, 1107

Crespo-Chacón, I., Montes, D., García-Alvarez, D., et al. 2006, A\&A, 452, 987

Demory, B., Ségransan, D., Forveille, T., et al. 2009, in AIP Conf. Ser. 1094, ed.

E. Stempels, 792

Dorman, B., Nelson, L. A., \& Chau, W. Y. 1989, ApJ, 342, 1003

Frogel, J. A., Kleinmann, D. E., Kunkel, W., Ney, E. P., \& Strecker, D. W. 1972, PASP, 84, 581

Fuhrmeister, B., Schmitt, J. H. M. M., \& Wichmann, R. 2004, A\&A, 417, 701

Fuhrmeister, B., Schmitt, J. H. M. M., \& Hauschildt, P. H. 2005, A\&A, 436, 677

Fuhrmeister, B., Liefke, C., Schmitt, J. H. M. M., \& Reiners, A. 2008, A\&A, 487, 293

Fuhrmeister, B., Schmitt, J. H. M. M., \& Hauschildt, P. H. 2010, A\&A, 511, A83

Gabriel, A. H., \& Jordan, C. 1969, MNRAS, 145, 241

Grevesse, N., \& Sauval, A. J. 1998, Space Sci. Rev., 85, 161

Güdel, M., Audard, M., Skinner, S. L., \& Horvath, M. I. 2002, ApJ, 580, L73

Güdel, M., Audard, M., Reale, F., Skinner, S. L., \& Linsky, J. L. 2004, A\&A, 416,713

Gunn, A. G., Doyle, J. G., Mathioudakis, M., Houdebine, E. R., \& Avgoloupis, S. 1994, A\&A, 285, 489

Haisch, B. M., Harnden, Jr., F. R., Seward, F. D., et al. 1980, ApJ, 242, L99

Haisch, B. M., Linsky, J. L., Bornmann, P. L., et al. 1983, ApJ, 267, 280

Haisch, B. M., Butler, C. J., Foing, B., Rodono, M., \& Giampapa, M. S. 1990, A\&A, 232, 387

Haisch, B., Strong, K. T., \& Rodono, M. 1991, ARA\&A, 29, 275

Haisch, B., Antunes, A., \& Schmitt, J. H. M. M. 1995, Science, 268, 1327

Haisch, B., Kashyap, V., Drake, J. J., \& Freeman, P. 1998, A\&A, 335, L101

Hauschildt, P. H., Allard, F., \& Baron, E. 1999, ApJ, 512, 377

Hawley, S. L., \& Fisher, G. H. 1992, ApJS, 78, 565

Hilton, E. J., West, A. A., Hawley, S. L., \& Kowalski, A. F. 2010, AJ, 140, 1402
Johns-Krull, C. M., \& Valenti, J. A. 2000, in Stellar Clusters and Associations: Convection, Rotation, and Dynamos, ed. R. Pallavicini, G. Micela, \& S. Sciortino, ASP Conf. Ser., 198, 371

Kowalski, A. F., Hawley, S. L., Holtzman, J. A., Wisniewski, J. P., \& Hilton, E. J. 2010a, ApJ, 714, L98

Kowalski, A. F., Hawley, S. L., Holtzman, J. A., Wisniewski, J. P., \& Hilton, E. J. 2010b [arXiv: 1010.0452v1]

Moore, C. E. 1972, Nat. Stand. Ref. Data Ser., 40

Ness, J.-U., \& Wichmann, R. 2002, Astron. Nachr., 323, 129

Neupert, W. M. 1968, ApJ, 153, L59

Osten, R. A., Hawley, S. L., Allred, J. C., Johns-Krull, C. M., \& Roark, C. 2005a, ApJ, 621, 398

Osten, R. A., Hawley, S. L., Allred, J. C., Johns-Krull, C. M., \& Roark, C. 2005b, ApJ, 621, 398

Parker, E. N. 1955, ApJ, 122, 293

Piskunov, N. E., \& Valenti, J. A. 2002, A\&A, 385, 1095

Pradhan, A. K., \& Shull, J. M. 1981, ApJ, 249, 821

Reale, F. 2007, A\&A, 471, 271

Reale, F., Betta, R., Peres, G., Serio, S., \& McTiernan, J. 1997, A\&A, 325, 782

Reale, F., Güdel, M., Peres, G., \& Audard, M. 2004, A\&A, 416, 733

Reid, I. N., Burgasser, A. J., Cruz, K. L., Kirkpatrick, J. D., \& Gizis, J. E. 2001, AJ, 121,1710

Reiners, A., \& Basri, G. 2006, ApJ, 644, 497

Reiners, A., \& Basri, G. 2007, ApJ, 656, 1121

Reiners, A., \& Basri, G. 2008, A\&A, 489, L45

Reiners, A., \& Basri, G. 2010, ApJ, 710, 924

Reiners, A., Schmitt, J. H. M. M., \& Liefke, C. 2007, A\&A, 466, L13

Robrade, J., \& Schmitt, J. H. M. M. 2005, A\&A, 435, 1073

Robrade, J., Poppenhaeger, K., \& Schmitt, J. H. M. M. 2010, A\&A, 513, A12

Serio, S., Reale, F., Jakimiec, J., Sylwester, B., \& Sylwester, J. 1991, A\&A, 241, 197

Short, C. I., \& Doyle, J. G. 1998, A\&A, 336, 613

Shulyak, D., Reiners, A., Wende, S., et al. 2010, A\&A, 523, A37

Smith, R. K., Brickhouse, N. S., Liedahl, D. A., \& Raymond, J. C. 2001, in Spectroscopic Challenges of Photoionized Plasmas, ed. G. Ferland, \& D. W. Savin, ASP Conf. Ser., 247, 161

Stelzer, B., Schmitt, J. H. M. M., Micela, G., \& Liefke, C. 2006, A\&A, 460, L35

Švestka, Z. 1972, ARA\&A, 10, 1

van Leeuwen, F. 2007, A\&A, 474, 653

Voges, W., Aschenbach, B., Boller, T., et al. 1999, A\&A, 349, 389

Wargelin, B. J., \& Drake, J. J. 2002, ApJ, 578, 503

West, A. A., \& Hawley, S. L. 2008, PASP, 120, 1161 